INTER NATIONAL MONETARY FUND
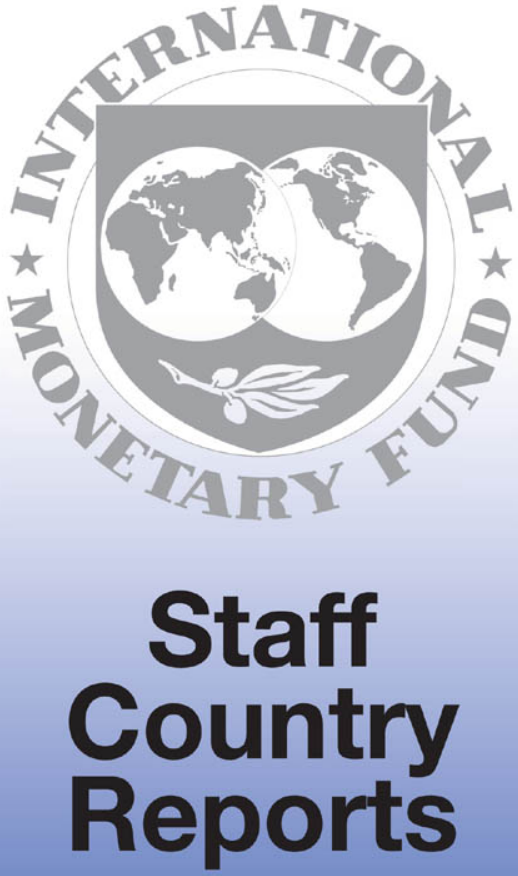


\section{Cape Verde: Second Review Under the Policy Support Instrument and Request for Waivers of Nonobservance of Assessment Criteria-Staff Report; Staff Supplements; Press Release on the Executive Board Discussion; and Statement by the Executive Director for Cape Verde.}

In the context of the second review under the Policy Support Instrument and request for waivers of nonobservance of assessment criteria, the following documents have been released and are included in this package:

- $\quad$ The staff report for the second review under the Policy Support Instrument and request for waivers of nonobservance of assessment criteria, prepared by a staff team of the IMF, following discussions that ended on November 29, 2011, with the officials of Cape Verde on economic developments and policies. Based on information available at the time of these discussions, the staff report was completed on January 13, 2012. The views expressed in the staff report are those of the staff team and do not necessarily reflect the views of the Executive Board of the IMF.

- Staff supplements on a Debt Sustainability Analysis and an Informational Annex.

- $\quad$ A Press Release summarizing the views of the Executive Board as expressed during its January 30, 2012 discussion of the staff report that completed the request and/or review.

- $\quad$ A statement by the Executive Director for Cape Verde.

The document listed below has been or will be separately released.

Letter of Intent sent to the IMF by the authorities of Cape Verde*

*Also included in the Staff Report

The policy of publication of staff reports and other documents allows for the deletion of market-sensitive information.

Copies of this report are available to the public from

International Monetary Fund • Publication Services

$70019^{\text {th }}$ Street, N.W. • Washington, D.C. 20431

Telephone: (202) 623-7430 • Telefax: (202) 623-7201

E-mail: publications@imf.org Internet: http://www.imf.org

\section{International Monetary Fund Washington, D.C.}


This page intentionally left blank

(C)International Monetary Fund. Not for Redistribution 


\section{INTERNATIONAL MONETARY FUND}

\section{CAPE VERDE}

\section{Second Review Under the Policy Support Instrument and Request for Waivers of Nonobservance of Assessment Criteria}

Prepared by the African Department

(In consultation with other departments)

\section{Approved by Sharmini Coorey and Claire Waysand}

January 13,2012

- $\quad$ Fund relations: A new 15-month PSI program was approved by the Executive Board on November 22, 2010. The first PSI review was completed by the Executive Board on a lapse-of-time basis on July 27, 2011.

- $\quad$ Staff team: Ms. Stotsky (head), Mr. Mu, Mr. Rodrigues Bastos, and Ms. Jin (all AFR). Mr. Sembene (OED) participated in the discussions.

- Discussions: Held during November 16-29, 2011. The mission met with Prime Minister Jose Maria Neves, Minister of Finance and Planning Cristina Duarte, Central Bank Governor Carlos de Burgo, other government officials, and representatives of civil society, development partners, and the private sector.

- $\quad$ Review: The end-September targets were missed for the net domestic assets (NDA) and net international reserves (NIR) of the Bank of Cape Verde (BCV). The authorities met or made substantial progress on all seven structural benchmarks. Staff recommends completion of the second review and waivers for the nonobservance of end-September NDA and NIR targets based on corrective actions taken by the authorities.

- $\quad$ Exchange rate regime: Conventional peg to the euro. 


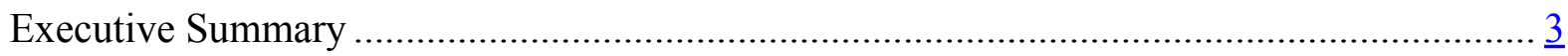

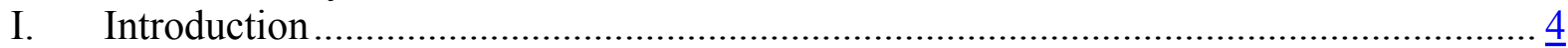

II. Recent Developments and Economic Outlook ............................................................ 4

III. Program Performance...............................................................................................

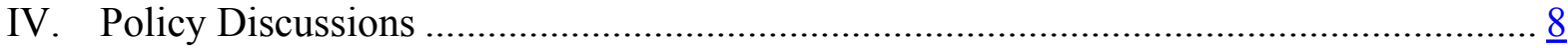

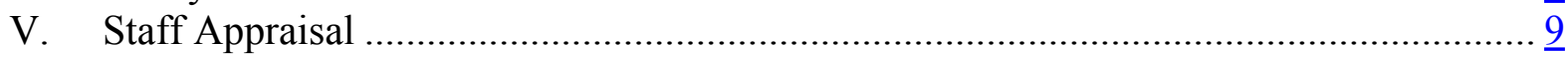

\section{Tables}

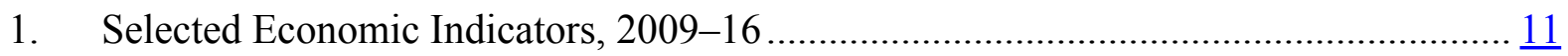

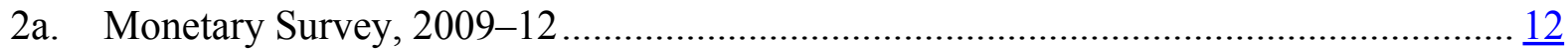

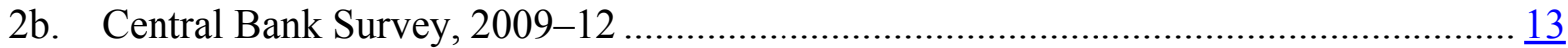

2c. Deposit Money Bank Survey, 2009-12 …………................................................. $\frac{13}{13}$

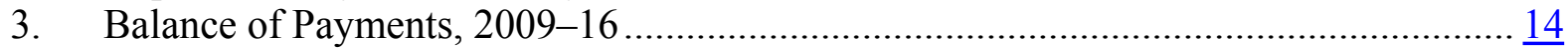

4. Financial Soundness of the Banking Sector........................................................ $\frac{15}{16}$

5a. Fiscal Operations of the Central Government, 2009-16........................................... $\frac{16}{17}$

5b. Fiscal Operations of the Central Government, 2009-16......................................... $\frac{17}{18}$

6. Quantitative Assessment Criteria and Indicative Targets for 2010-11 Under the PSI.. 18

7. Structural Benchmarks for 2010-11 ………........................................................... 19

\section{Figures}

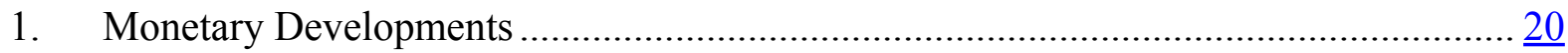

2. External Sector Developments .........................................................................

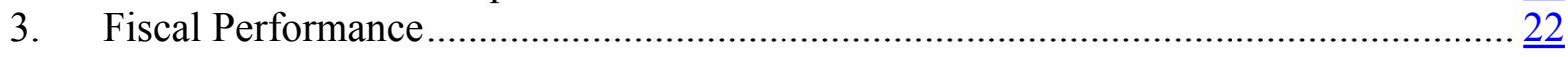

\section{Box}

1. Updated Assessment of the Exchange Rate …………….......................................... $\underline{5}$

\section{Appendices}

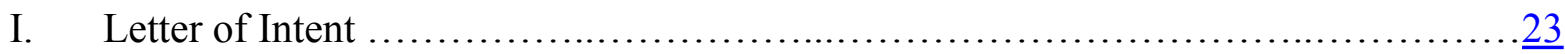

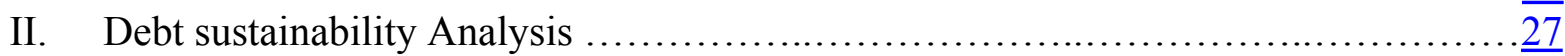

\section{Annexes}

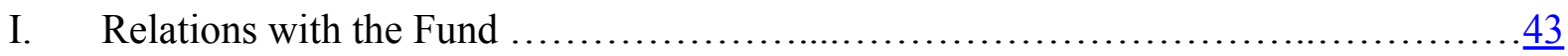

II. Joint IMF-World Bank Management Action Plan-Implementation Matrix ............. 46

III. Statistical Issues ............................................................... 


\section{EXECUTIVE SUMMARY}

- Growth is slowing while inflation has risen. Growth is expected to have eased to about 5 percent in 2011, reflecting the unsettled European economic and financial conditions. Consumer price inflation rose to 4.5 percent in 2011 (year-on-year), reflecting global food and fuel price shocks. Core inflation remains subdued.

- $\quad$ Program performance was mixed. End-September 2011 assessment criteria for NDA and NIR of the BCV were missed, reflecting external shocks to food and fuel prices and euro area disarray, and an accommodative monetary stance. However, by end-December 2011, the authorities had tightened the fiscal and monetary stances, and had significantly reduced the gaps with end-September program targets. The authorities met or made substantial progress on all seven structural benchmarks.

The fiscal target for calendar year 2011 was met, but further fiscal tightening is needed in 2012. Tax revenues held up well, but there were shortfalls in nontax revenue, in addition to capital transfers. The spending execution was held below the budget. The authorities need to scale back the fiscal deficit in 2012 and the medium term to ensure a sustainable fiscal position. Net domestic financing of the central government should decline to avoid crowding out private sector credit and lessen pressure on the balance of payments.

- Monetary conditions began tightening after mid-2011. The BCV adopted a more restrictive monetary stance by broadening and raising reserve requirements, restarting open market operations to absorb escudo, and raising intervention interest rates. Money growth has declined, in the face of a weak balance of payments. The real exchange rate remains broadly in line with fundamentals.

- $\quad$ Financial soundness indicators are generally favorable; however, vulnerabilities in the financial sector are increasing. The sharp slowdown in construction poses risks to the domestic banking system. Supervision of both the onshore and offshore financial sectors will need to be especially vigilant in the period ahead.

- The risks to Cape Verde's economic outlook are rising, with the increased financial disarray in Europe. Cape Verde needs to adhere to tight fiscal and monetary policies, while pressing ahead on structural reforms to safeguard the peg and strengthen competitiveness and fiscal sustainability. 


\section{INTRODUCTION}

1. The 15-month PSI program supported the government's policy goals. Its objectives included reinforcing macroeconomic stability, while accommodating some widening of the fiscal deficit for expanded capital investments.

2. The economy has performed well over the last decade. Cape Verde made noticeable progress towards meeting the Millennium Development Goals. Per capita income has reached over US $\$ 3,000$. The prime minister is currently serving his third consecutive term.

\section{RECENT DEVELOPMENTS AND ECONOMIC OUTLOOK}

\section{Macroeconomic performance is solid but has weakened.}

- GDP growth is projected to have slowed to about 5 percent in 2011, reflecting the negative external environment (Table 1). Tourism and public investments have provided some continuing momentum (Text Figure 1). Confidence indicators in key sectors present a mixed picture (Text Figure 2). ${ }^{1}$

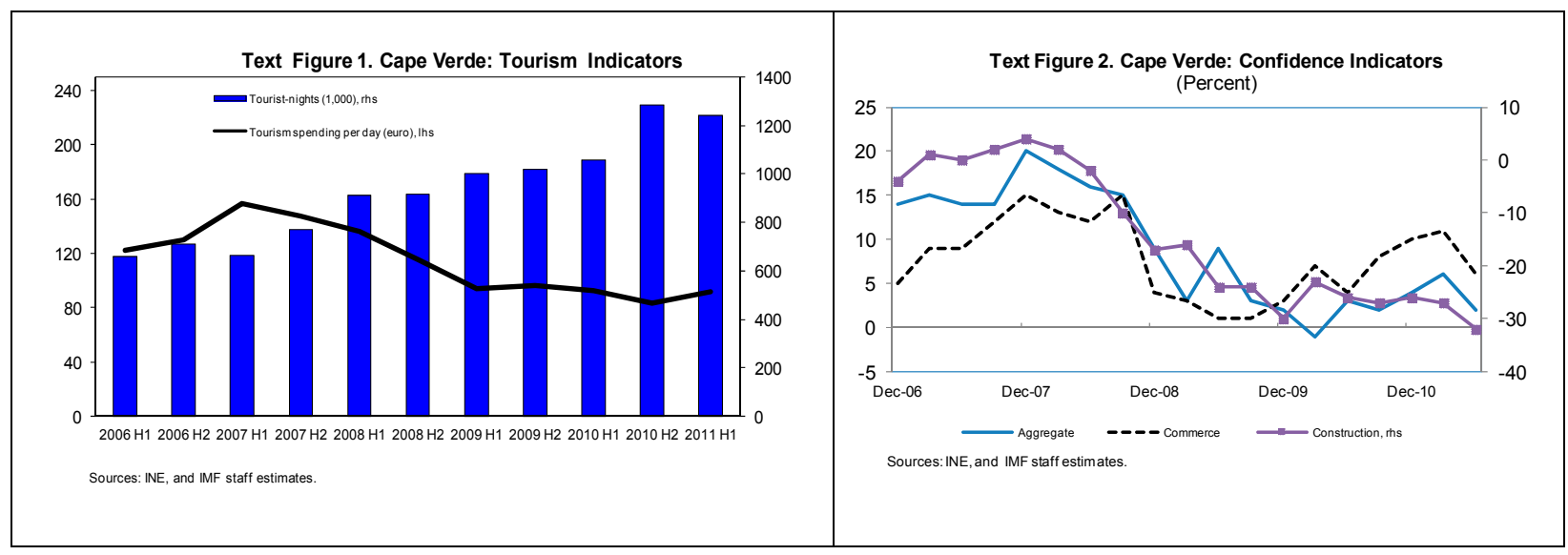

- $\quad$ Consumer price inflation rose to about 4.5 percent in 2011 (year-on-year), reflecting global food and fuel price shocks. Core inflation remains subdued.

4. Monetary conditions were accommodative in early 2011, but the BCV tightened its monetary policy stance after mid-year. The BCV's NDA at end-September 2011 exceeded its target by a significant margin, reflecting the weak external environment and an accommodative monetary stance, in particular, the running down of maturing central bank bills (Tables 2a, b, and c, and 3 and Figures 1 and 2). By mid-year, the BCV began taking

\footnotetext{
${ }^{1}$ The confidence indicators are produced by the National Statistics Institute, based on qualitative surveys distributed to employers inquiring about their economic situation and outlook. The numbers reflect the difference between extreme positive and negative responses as a percentage of total responses.
} 
actions to contain reserve and broad money growth. It extended reserve requirements to government deposits in July 2011, with modest effect; restarted regular auctions to absorb liquidity in November; and raised the reserve requirement on deposit liabilities by two percentage points to 18 percent and its policy rate by 150 basis points to 5.75 percent on January 1,2012 . The real effective exchange rate (REER) remains broadly in line with its fundamentals (Box 1).

\section{Box 1. Updated Assessment of the Exchange Rate}

This box reports the staff's updated assessment of Cape Verde's REER, which shows that the escudo is still broadly aligned with its fundamentals in the medium term (taken here as 2016). Using the September 2011 World Economic Outlook (WEO) database, the same three approaches used in the 2010 Article IV consultation staff report (IMF Country Report No. 10/349) were applied. The results also point to a somewhat more depreciated escudo compared to the last assessment.

Assessment of the Alignment of the Escudo

\begin{tabular}{lccc}
\hline & ERER & MB & ES \\
\cline { 2 - 4 } Current account norm (percent of GDP) & $\ldots$ & -9.4 & -7.9 \\
Current account gap (percent of GDP) & $\ldots$ & 1.6 & 0.1 \\
Misalignment estimate and 90 percent & -9.6 & -4.2 & -0.2 \\
confidence interval $^{1}$ & $(-27.8,20.9)$ & $(-25.7,17.3)$ & \\
\hline
\end{tabular}

Source: IMF staff estimates and projections

$1+$ means overvaluation, percent

The equilibrium real exchange rate (ERER) approach calculates the REER norm for 2016 based on a panel regression. Explanatory variables in the regression were 1973-2010 fundamentals of countries in the WEO database (i.e., relative productivity; terms of trade; government consumption; net foreign assets (NFA); aid inflows; and remittances). The REER norm was then calculated using the coefficients estimated from the regression and the WEO forecast of Cape Verde's 2016 fundamentals. Compared to the REER norm, Cape Verde's REER (assumed constant in the medium term) would be undervalued by 9.6 percent, with the 90 percent confidence interval ranging from a 27.8 percent undervaluation to a 20.9 percent overvaluation.

The macro-balance (MB) approach uses a panel regression similar to that of the ERER approach to calculate the 2016 current account balance (CAB) norm, instead of the REER norm, with a different set of fundamentals (i.e., relative old age dependency; relative population growth; relative income; relative income growth; relative fiscal balance; oil trade balance; initial NFA; aid inflows; and remittances). The gap between the WEO forecast of the $2016 \mathrm{CAB}$ and the CAB norm is 1.6 percent of GDP in 2016. Assuming an import elasticity of 0.92 and an export elasticity of -0.71 (as estimated in Isard and Faruqee, 1998), this gap implies an exchange rate undervaluation of 4.2 percent with the confidence interval $(-25.7,17.3)$.

The external sustainability (ES) approach directly assesses the gap between the WEO forecast of the $2016 \mathrm{CAB}$ and a CAB norm that would stabilize the NFA of Cape Verde at its 2010 level. Using the same trade elasticities as in the MB approach, the gap of 0.1 percent of GDP implies an exchange rate undervaluation of 0.2 percent.

Overall, the results suggest that the escudo is modestly undervalued, though the confidence intervals produce a mixed picture. Some limitations of the above approaches should be taken into account. For example, because of the scarcity of pricing data on tourism, the country's main export industry, the terms of trade variable was estimated using certain goods price indices as proxies, which may not fully capture the impact of exogenous price changes on the current account. 
5. Financial soundness indicators are generally favorable (Table 4). Although there has been some increase in nonperforming loans, they remain modest. A key vulnerability is the prospect of rising construction and real estate-related loan impairment. A second is a slowing of growth of sizeable emigrant deposits in domestic banks and shifting portfolio choices of large domestic depositors. In addition, the offshore sector is undergoing transition in ownership and its role in the economy.

6. Cape Verde is confronting a significantly worse external environment. Despite growth of tourism, fish exports, and private remittances, the sharp commodity price shock and rise of capital imports led to a sizeable widening of the current account deficit in 2011, and in combination with a shortfall in capital transfers and weaker foreign direct investment, the overall balance of payments turned sharply negative. The deficit was estimated at about 37 million euros, and reserve coverage is estimated to have ended the year at 3 months of imports.

7. The 2011 fiscal deficit was below the budget. Tax revenues held up well, but there were shortfalls in nontax revenue, in addition to capital transfers. The spending execution was held below the budget, to offset lower revenues, and to reduce domestic borrowing to help monetary adjustment (Tables $5 \mathrm{a}$ and $\mathrm{b}$ and Figure 3 ).

\section{The near-term outlook is fraught with risks from the financial stress and} increased possibility of recession in the euro area. The authorities need to contain credit expansion and minimize domestic budget financing, until import growth slows and the balance of payments improves. Reserves appear relatively low, at three months of import coverage, given Cape Verde's exposed position and its exchange rate peg. Enhanced monetary and fiscal policy coordination would help in finding the right balance between fiscal restraint and interest rate increases to contain credit growth while ensuring that reserves are built up over the medium term. If the euro area slowdown turns out to be protracted and the European financial system remains weak, foreign investment and growth in Cape Verde are likely to be dampened over the medium term.

\section{Program Performance}

9. Program performance under this 15-month PSI was mixed; reserve coverage declined in the face of significant external shocks; and the authorities' policy response was delayed. Two of eight quantitative assessment criteria for end-September 2011 were missed (Table 6). ${ }^{2}$ Although the first review was completed in July 2011 with waivers granted for the missed assessment criteria on the BCV's NDA and NIR targets because of what were seen as temporary and minor factors, the exogenous shocks to the balance of payments persisted and intensified. Two significant external shocks from food and fuel price increases

\footnotetext{
${ }^{2}$ In December 2011, the African Development Bank disbursed a quasi-concessional budget support loan of 15 million euros, which fell within the limit for such loans agreed in the PSI.
} 
and euro area financial disarray led to a continued weak balance of payments, ${ }^{3}$ accommodated by an unwinding of central bank bills (Text table). Some delays in the receipt of foreign assistance to finance the budget's scaling up of infrastructure spending, which had been absorbed into program targets, put further pressure on reserves and ran down government deposits in the BCV. As a consequence, the second review's end-September BCV NDA and NIR targets were missed by margins of 2.0 and 4.1 percent of GDP, respectively.

Text Table: Cape Verde's NDA and NIR Targets

(Flow from January 1, 2011 to indicated date)

\begin{tabular}{|c|c|c|c|c|c|c|c|c|c|}
\hline & \multicolumn{3}{|c|}{ End March } & \multicolumn{4}{|c|}{ End September } & \multicolumn{2}{|c|}{ End December } \\
\hline & $\begin{array}{l}\text { Assessment } \\
\text { criteria }\end{array}$ & $\begin{array}{l}\text { Adjusted } \\
\text { assessment } \\
\text { criteria }\end{array}$ & Actual & $\begin{array}{l}\text { Assessment } \\
\text { criteria }\end{array}$ & $\begin{array}{l}\text { Adjusted } \\
\text { assessment } \\
\text { criteria }\end{array}$ & Actual & Gap & Actual & $\begin{array}{l}\text { Change } \\
\text { since } \\
\text { September }\end{array}$ \\
\hline \multicolumn{10}{|c|}{ (Billions of Cape Verde escudos) } \\
\hline NDA & 0.3 & 2.4 & 3.2 & 0.8 & 3.6 & 6.6 & 3.0 & 1.8 & -4.8 \\
\hline \multicolumn{10}{|c|}{ (Millions of euros) } \\
\hline NIR & 6.6 & -12.8 & -22.7 & 19.8 & -5.5 & -61.4 & -55.8 & -34.2 & 27.2 \\
\hline
\end{tabular}

\section{Despite the missed targets, by end-December 2011, the authorities had taken} significant fiscal and monetary measures to bring the monetary program back on track. The government reduced spending well below budgeted amounts, while preserving the social safety net. The BCV restricted domestic credit growth through extending reserve requirements and restarting regular open market operations. The tightened policy stances reduced BCV NDA growth by end-December to close about two-thirds of the gap with the missed end-September NDA target. The BCV made significant progress on reversing the reserve losses, ending the year with a reserve loss of about one-half of a month of import coverage.

\section{The authorities met or made substantial progress on all seven structural}

benchmarks (Table 7). The authorities met five benchmarks, a few after the end-September deadline. The Minister of Finance and BCV Governor signed a memorandum on monetary and fiscal cooperation and also a memorandum on establishing a financial stability committee; the Treasury completed the development of fungible bonds and extended the contingent liabilities report on the state-owned enterprises to encompass cross-enterprise debt; and the authorities completed a medium-term debt strategy (MTDS). The two outstanding benchmarks are for

\footnotetext{
${ }^{3}$ Higher food and fuel prices accounted for 34 million euros, lower current transfers 13 million euros, and lower foreign direct investment 21 million euros, of the gap compared to earlier estimates.
} 
submission to the parliament of the financial system and tax laws, which are being redrafted with IMF assistance and are expected to be submitted to the legislature in early $2012 .{ }^{4}$

\section{Policy Discussions}

\section{The authorities agreed that it is essential to sustain the tightened fiscal and} monetary stances, which will support the rebuilding of reserves and slow the accumulation of debt. In addition to intensifying its efforts to restrain credit growth in late 2011, the BCV, in January 2012, raised reserve requirements and increased the intervention interest rate to sustain progress on rebuilding reserves, thus demonstrating firm commitment to a prudent monetary stance in view of intensified external risks.

\section{The authorities are sustaining a tighter fiscal stance in $\mathbf{2 0 1 2}$ and are pursuing a} medium-term fiscal scenario of gradual deficit reduction. Following the adoption of the 2012 budget, the authorities agreed to contain spending, including fiscal transfers, and net domestic borrowing to a minimal level in the first half of this year. Over the medium-term, staff projects a peaking and then gradual reduction of debt ratios, which is broadly aligned with the government's plan, though staff preferred a faster reduction of debt. Revenues as a percent of GDP are projected to fall owing to a decline of official grants, partly offset by higher own revenues as tax reforms are implemented in 2013. The government is expected to continue to pursue a tight control of current expenditures.

14. The mission made progress on developing a consolidated view of the government, including the social security fund, parastatals, and local governments. The social security fund, with a surplus of about 2-3 percent of GDP per year, has accumulated significant assets, mostly in bank deposits and government securities. The authorities agreed on the need to put in place a proper framework for managing and valuing the social security fund's assets. The mission discussed the need to amend the tax law to eliminate any loopholes that encourage inefficient shifting of money between the fund and commercial banks. Staff encourages the authorities to reduce the fund's tax rate on wage income, which is relatively high for a lower middle-income country and discourages formal sector employment, while restructuring the benefit side to ensure the fund's long-term fiscal solvency. The local government deficit appears to be modest. The authorities are working toward consolidating the broader public sector.

\section{Progress on state-owned enterprise reform has been modest, and staff urged the} authorities to redouble their efforts. The government is reforming the two loss-making parastatals, Electra (the electricity and water company) and TACV (the national airline), with the idea of eventual privatization. It is essential that the unit costs of electricity and water fall to more competitive levels.

\footnotetext{
${ }^{4}$ The change of government in mid-summer necessitated some delay in the completion of these benchmarks.
} 


\section{Cape Verde's public debt remains sustainable, but a worsening global}

environment has accentuated macroeconomic vulnerabilities. The staff emphasized the importance of reducing more quickly the fiscal deficit trajectory by reducing public investment borrowing and strengthening own revenues. Staff's debt sustainability analysis suggests that the public debt burden remains sustainable, but that exogenous shocks could cause some debt indicators to breach thresholds. A particular concern is the relatively high level of private sector external debt, facilitated by Cape Verde's peg. The MTDS supports the continuation of the present financing strategy, while flagging the importance of improving domestic debt markets as access to concessional foreign financing declines. ${ }^{5}$

17. In view of increasing financial sector vulnerabilities, the authorities agreed on the importance of continuing to strengthen banking supervision in both onshore and offshore sectors. The authorities indicated their intention to use the new financial framework and banks laws to continue strengthening financial supervision and with the establishment of a financial stability committee, mitigate financial sector risks.

18. Growth needs to be more inclusive. Cape Verde has a double-digit unemployment rate that has shown no substantial improvement over the last decade despite strong growth performance. Authorities are committed to diversifying economic activity, which should lead to new job opportunities. In addition, efforts to align labor market incentives and skills supply are important to improve growth inclusiveness. The social safety net in place provides good protection to the poor and vulnerable.

\section{The mission discussed possible parameters for renewed PSI engagement, should} the authorities seek it. A key objective would entail reducing the fiscal deficit trajectory to ensure that the debt to GDP ratio begins to decline by 2013 or 2014 . While the scaling up of infrastructure spending was agreed to in the PSI programs, the worsened external environment gives cause to bring down the debt to GDP ratio more quickly than had been envisioned. In addition, the relatively modest goal of the concluding PSI for accumulation of reserves would need to be strengthened to at least four months of import coverage by the end of a new PSI.

\section{Staff Appraisal}

20. Cape Verde's economy remains on an even keel but the authorities' response to a significant weakening of its balance of payments in 2011, in the face of two external shocks, was delayed. The economy continues to grow at a moderate rate and core inflation is contained. Nonetheless, the rising risks in the euro zone and slowing global growth emphasize the need for the authorities to react more quickly to shocks, and to redouble their commitment to raising reserve coverage to safeguard the peg and to reducing the public debt to a more prudent level.

\footnotetext{
${ }^{5}$ The mission encouraged the authorities to apply the technical capacity built during the MTDS to strengthen debt management analysis.
} 
21. Staff welcomes the authorities' tightening of the fiscal stance and intention to adhere to a tight stance in 2012. Fiscal policy is the main anchor of Cape Verde's exchange rate regime. In the short-term, reducing net domestic financing of the budget will lessen pressure on the balance of payments and leave room for private sector credit growth. In the medium-term, the authorities should significantly scale back the overall fiscal deficit to bolster medium-term sustainability, in view of slower domestic growth and debt rollover risk, when concessional debt comes due. It is essential that the authorities broaden the tax base and sustain efforts to control current expenditures.

22. A tightened monetary policy stance should help to stabilize reserve coverage. The authorities should strive to leave room for credit to the private sector, even within a constrained external and domestic financial environment. Improved coordination between fiscal and monetary policies will lessen the costs of liquidity management.

23. Supervision of both the onshore and offshore financial sectors will need to be especially vigilant in the period ahead. Staff welcomes the completion of the financial stability committee framework, and encourages its speedy implementation and the completion of updated financial system legislation. The authorities should continue strengthening supervision of the financial system, with development partner assistance.

24. The authorities should press ahead with restructuring state-owned enterprises. Cape Verde's growth prospects depend on sustaining economic competitiveness, especially in the tourist sector. Reduced costs and better access to key utilities and an improved quality and flexibility of the labor force are essential.

25. Staff recommends the completion of the PSI second review and granting of waivers for the nonobservance of end-September targets on the BCV's NDA and NIR. The authorities' corrective actions taken to tighten the fiscal and monetary stances have started to rebuild reserves and brought domestic credit growth close to program objectives by endDecember 2011. In addition, the authorities completed or are soon to complete all structural benchmarks. 
Table 1. Cape Verde: Selected Economic Indicators, 2009-16

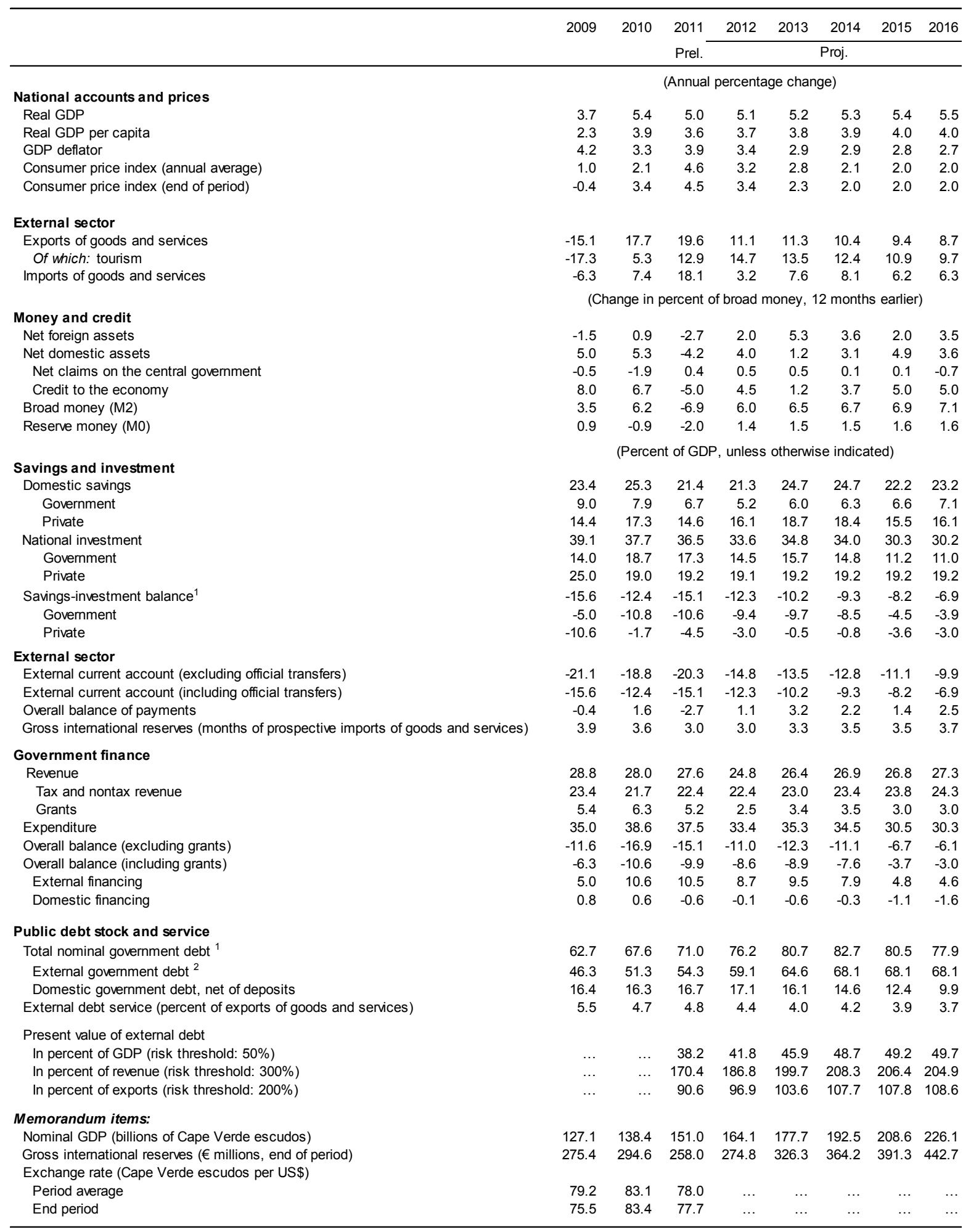

Sources: Cape Verdean authorities; and IMF staff estimates and projections.

${ }^{1}$ Net of central government deposits.

${ }^{2}$ Excluding claims on the offshore Trust Fund. 
Table 2a. Cape Verde: Monetary Survey, 2009-12

\begin{tabular}{|c|c|c|c|c|c|c|c|}
\hline & 2009 & 2010 & $\begin{array}{r}2011 \\
\text { End Mar }\end{array}$ & End June & End Sept & $\begin{array}{r}2011 \\
\text { Prel. } \\
\text { End Dec }\end{array}$ & $\begin{array}{r}2012 \\
\text { Proj. }\end{array}$ \\
\hline & \multicolumn{7}{|c|}{ (Millions of Cape Verde escudos, unless otherw ise indicaded) } \\
\hline Net foreign assets & 27,414 & 28,379 & 26,222 & 20,465 & 20,003 & 25,352 & 27,399 \\
\hline $\begin{array}{l}\text { Foreign assets } \\
\text { Of which: gross international reserves } \\
\text { Foreign liabilities }\end{array}$ & $\begin{array}{r}37,614 \\
30,371 \\
-10,200\end{array}$ & $\begin{array}{r}39,763 \\
32,486 \\
-10,422\end{array}$ & $\begin{array}{r}36,375 \\
29,948 \\
-10,153\end{array}$ & $\begin{array}{r}30,708 \\
25,113 \\
-10,243\end{array}$ & $\begin{array}{r}31,777 \\
25,689 \\
-11,774\end{array}$ & $\begin{array}{r}35,550 \\
28,450 \\
-10,197\end{array}$ & $\begin{array}{r}37,476 \\
30,305 \\
-10,077\end{array}$ \\
\hline $\begin{array}{l}\text { Net domestic assets } \\
\text { Net domestic credit } \\
\text { Net claims on general government (net) } \\
\text { Investment in TCMFs }{ }^{1} \\
\text { Net claims on the central government } \\
\text { Net claims on local government } \\
\text { Credit to the economy } \\
\text { Other items (net) }\end{array}$ & $\begin{array}{r}77,893 \\
97,821 \\
18,599 \\
11,038 \\
7,403 \\
1,052 \\
79,222 \\
-19,928\end{array}$ & $\begin{array}{r}83,458 \\
103,907 \\
17,636 \\
11,584 \\
5,426 \\
1,544 \\
86,271 \\
-20,450\end{array}$ & $\begin{array}{r}86,179 \\
109,073 \\
21,269 \\
11,000 \\
8,288 \\
1,981 \\
87,804 \\
-22,894\end{array}$ & $\begin{array}{r}90,491 \\
113,079 \\
22,156 \\
11,153 \\
8,859 \\
2,144 \\
90,923 \\
-22,587\end{array}$ & $\begin{array}{r}93,196 \\
116,371 \\
24,284 \\
11,505 \\
10,469 \\
2,309 \\
92,087 \\
-23,175\end{array}$ & $\begin{array}{r}78,735 \\
99,586 \\
18,887 \\
11,379 \\
5,807 \\
2,400 \\
80,699 \\
-20,852\end{array}$ & $\begin{array}{r}82,933 \\
105,599 \\
20,250 \\
11,550 \\
6,307 \\
2,613 \\
85,349 \\
-22,666\end{array}$ \\
\hline $\begin{array}{l}\text { Broad money (M2) } \\
\text { Narrow money (M1) } \\
\text { Currency outside banks } \\
\text { Demand deposits } \\
\text { Quasi-money }\end{array}$ & $\begin{array}{r}105,307 \\
43,014 \\
8,362 \\
34,652 \\
57,700\end{array}$ & $\begin{array}{r}111,838 \\
47,022 \\
8,299 \\
38,723 \\
60,382\end{array}$ & $\begin{array}{r}112,401 \\
45,601 \\
7,545 \\
38,057 \\
61,786\end{array}$ & $\begin{array}{r}110,957 \\
45,006 \\
7,198 \\
37,808 \\
61,184\end{array}$ & $\begin{array}{r}113,199 \\
43,496 \\
7,484 \\
36,012 \\
65,567\end{array}$ & $\begin{array}{r}104,087 \\
42,676 \\
6,892 \\
35,783 \\
57,286\end{array}$ & $\begin{array}{r}110,332 \\
45,236 \\
6,689 \\
38,547 \\
60,723\end{array}$ \\
\hline Foreign currency deposits & 4,593 & $\begin{array}{r}4,433 \\
\text { (Change in }\end{array}$ & $\begin{array}{r}5,013 \\
\text { ercent of }\end{array}$ & $\begin{array}{l}4,767 \\
\text { oroad mone }\end{array}$ & $\begin{array}{r}4,137 \\
\text { ey, } 12 \text { montr }\end{array}$ & $\begin{array}{l}4,126 \\
\text { earlier) }\end{array}$ & 4,373 \\
\hline Net foreign assets & -1.5 & 0.9 & -0.6 & -6.8 & -6.9 & -2.7 & 2.0 \\
\hline Net domestic assets & 5.0 & 5.3 & 7.1 & 11.2 & 10.7 & -4.2 & 4.0 \\
\hline Net domestic credit & 7.9 & 5.8 & 8.6 & 12.7 & 12.4 & -3.9 & 5.8 \\
\hline Net claims on the central government & -0.5 & -1.9 & 1.8 & 4.5 & 5.2 & 0.3 & 0.5 \\
\hline Credit to the economy & 8.0 & 6.7 & 6.8 & 8.2 & 7.2 & -5.0 & 4.5 \\
\hline Other items (net) & -2.9 & -0.5 & -1.5 & -1.4 & -1.7 & -0.4 & -1.7 \\
\hline Broad money (M2) & 3.5 & 6.2 & 6.5 & 4.4 & 3.8 & -6.9 & 6.0 \\
\hline Memorandum items: & & & & & & & \\
\hline Emigrant deposits/total deposits (percent) & 37.1 & 37.1 & 35.8 & 36.7 & 37.3 & 36.5 & 36.4 \\
\hline Excess reserves/total deposits (percent) & 1.8 & 0.9 & 0.8 & -2.0 & -1.2 & 0.9 & 0.9 \\
\hline Money multiplier (M2/M0) & 3.9 & 4.3 & 4.2 & 4.5 & 4.4 & 4.4 & 4.4 \\
\hline
\end{tabular}

Sources: Bank of Cape Verde; and IMF staff estimates and projections.

${ }^{1}$ TCMFs (Títulos Consolidados de Mobilização Financeira) are government bonds in euros maturing in 2018 and backed by a Trust Fund w hich is managed by the Banco de Portugal.

2 INPS: Cape Verde's National Pension Institute. 
Table 2b. Cape Verde: Central Bank Survey, 2009-12

(Millions of Cape Verde escudos, unless otherw ise indicated)

\begin{tabular}{|c|c|c|c|c|c|c|c|}
\hline & 2009 & 2010 & $\begin{array}{l}2011 \\
\text { End Mar }\end{array}$ & $\begin{array}{r}2011 \\
\text { End Jun }\end{array}$ & $\begin{array}{r}2011 \\
\text { End Sept }\end{array}$ & $\begin{array}{r}2011 \\
\text { Prel. } \\
\text { End Dec }\end{array}$ & $\begin{array}{c}2012 \\
\text { Proj. }\end{array}$ \\
\hline Net foreign assets & 27,962 & 29,902 & 27,298 & 22,545 & 23,053 & 25,912 & 27,965 \\
\hline Of which: net international reserves & 29,476 & 31,742 & 29,235 & 24,458 & 24,975 & 27,974 & 30,027 \\
\hline Foreign assets & 30,507 & 32,631 & 29,948 & 25,113 & 25,689 & 28,450 & 30,305 \\
\hline Of which: gross international reserves & 30,371 & 32,486 & 29,948 & 25,113 & 25,689 & 28,450 & 30,305 \\
\hline Foreign liabilities & $-2,545$ & $-2,729$ & $-2,651$ & $-2,568$ & $-2,635$ & $-2,537$ & $-2,340$ \\
\hline Net domestic assets & $-1,128$ & $-3,986$ & -820 & 2,064 & 2,617 & $-2,202$ & $-2,832$ \\
\hline Net domestic credit & 1,334 & $-1,356$ & 1,006 & 5,075 & 5,780 & 398 & -267 \\
\hline Investment in TCMFs & 4,605 & 4,858 & 4,936 & 5,090 & 5,442 & 5,279 & 5,200 \\
\hline Credit to central government (net) & $-1,431$ & $-4,158$ & $-1,824$ & $-2,971$ & $-1,174$ & $-3,408$ & $-2,908$ \\
\hline Credit to commercial banks (net) & $-2,939$ & $-3,363$ & $-3,394$ & 1,645 & 293 & $-2,012$ & $-3,100$ \\
\hline Credit to the economy & 1,099 & 1,308 & 1,288 & 1,311 & 1,220 & 538 & 541 \\
\hline Other items (net) & $-2,462$ & $-2,630$ & $-1,826$ & $-3,012$ & $-3,163$ & $-2,600$ & $-2,565$ \\
\hline Reserve money (M0) & 26,834 & 25,916 & 26,478 & 24,609 & 25,671 & 23,710 & 25,133 \\
\hline Currency issued & 10,190 & 10,190 & 9,098 & 8,946 & 8,872 & 7,920 & 8,691 \\
\hline Deposits of commercial banks & 16,984 & 15,726 & 17,380 & 15,663 & 16,799 & 15,790 & 16,958 \\
\hline \multicolumn{8}{|l|}{ Memorandum items: } \\
\hline Gross international reserves (millions of euros) & 275.4 & 294.6 & 271.6 & 227.8 & 233.0 & 258.0 & 274.8 \\
\hline Net international reserves (millions of euros) & 267.3 & 287.9 & 265.1 & 221.8 & 226.5 & 253.7 & 272.3 \\
\hline
\end{tabular}

Sources: Bank of Cape Verde; and IMF staff estimates and projections.

Table 2c. Cape Verde: Deposit Money Bank Survey, 2009-12

(Millions of Cape Verde escudos, unless otherw ise indicated)

\begin{tabular}{|c|c|c|c|c|c|c|c|}
\hline & 2009 & 2010 & $\begin{array}{l}2011 \\
\text { End Mar }\end{array}$ & $\begin{array}{r}2011 \\
\text { End Jun }\end{array}$ & $\begin{array}{r}2011 \\
\text { End Sept }\end{array}$ & $\begin{array}{r}2011 \\
\text { Prel. } \\
\text { End Dec }\end{array}$ & $\begin{array}{r}2012 \\
\text { Proj. }\end{array}$ \\
\hline $\begin{array}{l}\text { Net foreign assets } \\
\text { Foreign assets } \\
\text { Foreign liabilities }\end{array}$ & $\begin{array}{r}-548 \\
7,107 \\
-7,655\end{array}$ & $\begin{array}{r}-562 \\
7,132 \\
-7,693\end{array}$ & $\begin{array}{r}-1,076 \\
6,426 \\
-7,502\end{array}$ & $\begin{array}{r}-2,080 \\
5,595 \\
-7,675\end{array}$ & $\begin{array}{r}-3,050 \\
6,088 \\
-9,138\end{array}$ & $\begin{array}{r}-560 \\
7,100 \\
-7,660\end{array}$ & $\begin{array}{r}-566 \\
7,171 \\
-7,737\end{array}$ \\
\hline $\begin{array}{l}\text { Net domestic assets } \\
\text { Net domestic credit } \\
\text { Net claims on general government } \\
\text { Investment in TCMFs } \\
\text { Net claims on central government } \\
\text { Net claims on local government } \\
\text { Credit to the economy } \\
\text { Net claims on the Bank of Cape Verde } \\
\text { Other items (net) }\end{array}$ & $\begin{array}{r}97,490 \\
113,805 \\
15,425 \\
6,433 \\
8,834 \\
1,052 \\
78,123 \\
20,257 \\
-16,315\end{array}$ & $\begin{array}{r}104,096 \\
122,085 \\
16,937 \\
6,727 \\
9,584 \\
1,544 \\
84,963 \\
21,145 \\
-17,989\end{array}$ & $\begin{array}{r}105,932 \\
124,770 \\
18,157 \\
6,064 \\
10,113 \\
1,981 \\
86,516 \\
20,096 \\
-18,838\end{array}$ & $\begin{array}{r}105,839 \\
125,903 \\
20,037 \\
6,064 \\
11,830 \\
2,144 \\
89,612 \\
16,254 \\
-20,065\end{array}$ & $\begin{array}{r}108,766 \\
127,912 \\
20,016 \\
6,064 \\
11,643 \\
2,309 \\
90,868 \\
17,028 \\
-19,147\end{array}$ & $\begin{array}{r}97,755 \\
116,755 \\
17,015 \\
6,100 \\
9,215 \\
2,400 \\
80,161 \\
19,578 \\
-19,000\end{array}$ & $\begin{array}{r}104,208 \\
124,718 \\
17,958 \\
6,350 \\
9,215 \\
2,613 \\
84,808 \\
21,952 \\
-20,510\end{array}$ \\
\hline $\begin{array}{l}\text { Deposit liabilities to nonbank residents } \\
\text { Local currency deposits } \\
\text { Demand deposits } \\
\text { Time deposits } \\
\text { Foreign currency deposits }\end{array}$ & $\begin{array}{r}96,942 \\
92,349 \\
34,649 \\
57,700 \\
4,593\end{array}$ & $\begin{array}{r}103,534 \\
99,101 \\
38,719 \\
60,382 \\
4,433\end{array}$ & $\begin{array}{r}104,856 \\
99,843 \\
38,057 \\
61,771 \\
5,013\end{array}$ & $\begin{array}{r}103,759 \\
98,992 \\
37,808 \\
61,169 \\
4,767\end{array}$ & $\begin{array}{r}105,716 \\
101,579 \\
36,012 \\
65,552 \\
4,137\end{array}$ & $\begin{array}{r}97,195 \\
93,069 \\
35,783 \\
57,286 \\
4,126\end{array}$ & $\begin{array}{r}103,643 \\
99,270 \\
38,547 \\
60,723 \\
4,373\end{array}$ \\
\hline
\end{tabular}

Sources: Bank of Cape Verde; and IMF staff estimates and projections. 
Table 3. Cape Verde: Balance of Payments, 2009-16

(Millions of euros, unless otherwise indicated)

\begin{tabular}{|c|c|c|c|c|c|c|c|c|}
\hline & 2009 & 2010 & 2011 & 2012 & 2013 & 2014 & 2015 & 2016 \\
\hline & Act. & Act. & Prel. & Proj. & Proj. & Proj. & Proj. & Proj. \\
\hline Current account balance (including official transfers) & -180 & -156 & -207 & -183 & -164 & -162 & -155 & -142 \\
\hline Trade balance & -487 & -513 & -619 & -637 & -697 & -761 & -806 & -856 \\
\hline Exports, f.o.b. & 67 & 102 & 146 & 152 & 161 & 169 & 177 & 186 \\
\hline Imports, f.o.b. & -554 & -615 & -765 & -789 & -858 & -930 & -983 & $-1,041$ \\
\hline Services (net) & 115 & 155 & 204 & 255 & 310 & 360 & 406 & 450 \\
\hline Receipt & 344 & 381 & 431 & 490 & 554 & 620 & 687 & 752 \\
\hline Of which: tourism & 190 & 200 & 226 & 259 & 294 & 330 & 366 & 401 \\
\hline Payment & -228 & -226 & -227 & -235 & -244 & -260 & -281 & -302 \\
\hline Income (net) & -31 & -55 & -49 & -53 & -57 & -62 & -65 & -68 \\
\hline Credit & 16 & 11 & 9 & 10 & 11 & 12 & 14 & 15 \\
\hline Debit & -47 & -66 & -58 & -63 & -68 & -75 & -78 & -83 \\
\hline Government interest & -5 & -6 & -9 & -11 & -12 & -16 & -16 & -17 \\
\hline Interest by other sectors & -16 & -17 & -18 & -19 & -20 & -21 & -22 & -23 \\
\hline Income on direct investment and other income & -27 & -42 & -31 & -33 & -35 & -38 & -40 & -43 \\
\hline Current transfers (net) & 223 & 257 & 257 & 252 & 280 & 301 & 310 & 332 \\
\hline Government & 63 & 80 & 71 & 37 & 54 & 62 & 56 & 62 \\
\hline Private & 161 & 177 & 185 & 216 & 226 & 239 & 254 & 270 \\
\hline Capital and financial account (net) & 182 & 237 & 177 & 200 & 215 & 200 & 182 & 193 \\
\hline Capital account & 32 & 29 & 5 & 10 & 11 & 12 & 13 & 14 \\
\hline \multicolumn{9}{|l|}{ Financial account } \\
\hline Direct investment & 86 & 84 & 56 & 80 & 82 & 83 & 85 & 87 \\
\hline Portfolio investment & 4 & 0 & 1 & 2 & 2 & 2 & 2 & 2 \\
\hline Other investment & 60 & 124 & 114 & 108 & 120 & 103 & 81 & 90 \\
\hline Central government & 57 & 133 & 144 & 129 & 153 & 138 & 90 & 95 \\
\hline Disbursements & 75 & 150 & 163 & 146 & 170 & 155 & 108 & 112 \\
\hline Amortization & -18 & -17 & -19 & -17 & -17 & -17 & -18 & -18 \\
\hline Monetary authority & 2 & 1 & -5 & -2 & -1 & -1 & 4 & 9 \\
\hline Commercial banks & 8 & 6 & 0 & 0 & 0 & 0 & 0 & 0 \\
\hline Nonbank flows & -7 & -16 & -25 & -20 & -32 & -35 & -13 & -13 \\
\hline Of which: nonbank trade credits & 5 & 3 & 0 & 0 & 0 & 0 & 0 & 0 \\
\hline Errors and omissions ${ }^{1}$ & -7 & -61 & -6 & 0 & 0 & 0 & 0 & 0 \\
\hline Overall balance & -5 & 20 & -37 & 17 & 51 & 38 & 27 & 51 \\
\hline Financing & 5 & -20 & 37 & -17 & -51 & -38 & -27 & -51 \\
\hline Gross international reserves (- accumulation) & 4 & -20 & 37 & -17 & -51 & -38 & -27 & -51 \\
\hline Exceptional financing & 1 & -1 & 0 & 0 & 0 & 0 & 0 & 0 \\
\hline \multicolumn{9}{|l|}{ Memorandum items: } \\
\hline Current account (including official transfers, percent of GDP) & -15.6 & -12.4 & -15.1 & -12.3 & -10.2 & -9.3 & -8.2 & -6.9 \\
\hline Current account (excluding official transfers, percent of GDP) & -21.1 & -18.8 & -20.3 & -14.8 & -13.5 & -12.8 & -11.1 & -9.9 \\
\hline Overall balance (percent of GDP) & -0.4 & 1.6 & -2.7 & 1.1 & 3.2 & 2.2 & 1.4 & 2.5 \\
\hline Gross international reserves & 275 & 295 & 258 & 275 & 326 & 364 & 391 & 443 \\
\hline Months of current year's imports of goods and services & 4.2 & 4.2 & 3.1 & 3.2 & 3.6 & 3.7 & 3.7 & 4.0 \\
\hline Months of next year's imports of goods and services & 3.9 & 3.6 & 3.0 & 3.0 & 3.3 & 3.5 & 3.5 & 3.7 \\
\hline External public debt & 533 & 644 & 744 & 880 & 1,041 & 1,188 & 1,289 & 1,395 \\
\hline
\end{tabular}

Sources: Bank of Cape Verde; and IMF staff estimates and projections.

${ }^{1}$ Including banks' delays on trade credit reporting. 
Table 4. Cape Verde: Financial Soundness of the Banking Sector

(End-year; percent unless otherwise indicated)

\begin{tabular}{|c|c|c|c|c|c|c|c|}
\hline Indicator & 2005 & 2006 & 2007 & 2008 & 2009 & 2010 & Sep-11 \\
\hline \multicolumn{8}{|l|}{ Capital adequacy } \\
\hline Regulatory capital to risk-weighted assets & 12.1 & 11.1 & 11.4 & 12.0 & 11.4 & 15.6 & 14.5 \\
\hline Regulatory tier 1 capital to risk-weighted assets & 13.4 & 11.3 & 11.8 & 11.5 & 11.2 & 14.4 & 13.5 \\
\hline \multicolumn{8}{|l|}{ Asset quality ${ }^{1}$} \\
\hline Nonperforming loans to total loans & 6.3 & 3.9 & 13.5 & 10.0 & 3.9 & 4.0 & 6.9 \\
\hline Nonperforming loans net of provisions to capital & -2.5 & -1.7 & 91.1 & 42.2 & -15.2 & -14.6 & 3.9 \\
\hline Provisions to nonperforming loans & 104.9 & 104.1 & 35.0 & 53.1 & 143.5 & 145.2 & 93.0 \\
\hline \multicolumn{8}{|l|}{ Earnings and profitability } \\
\hline Return on assets & 0.5 & 1.1 & 1.6 & 1.0 & 0.7 & 0.7 & 0.4 \\
\hline Return on equity & 9.7 & 19.7 & 30.1 & 15.8 & 9.8 & 9.9 & 4.5 \\
\hline Interest margin to gross income & 62.7 & 67.2 & 71.3 & 68.0 & 74.9 & 77.0 & 77.3 \\
\hline Noninterest expenses to gross income & 61.2 & 54.5 & 46.1 & 57.3 & 67.0 & 67.0 & 67.8 \\
\hline \multicolumn{8}{|l|}{ Liquidity $^{2}$} \\
\hline Liquid assets to total assets & 46.2 & 18.3 & 17.7 & 16.6 & 16.4 & 12.5 & 13.6 \\
\hline Liquid assets to short-term liabilities & 170.2 & 46.1 & 42.9 & 43.8 & 49.2 & 37.7 & 43.9 \\
\hline
\end{tabular}

Source: Bank of Cape Verde.

${ }^{1}$ The NPL ratio for 2007-08 applied stricter loan classification criteria, where a loan was considered nonperforming if it is only 1 day overdue.

${ }^{2}$ Liquid assets include cash in vault and marketable securities. Short-term liabilities include demand deposits. 
Table 5a. Cape Verde: Fiscal Operations of the Central Government, 2009-16

(Millions of Cape Verde escudos, unless otherwise indicated)

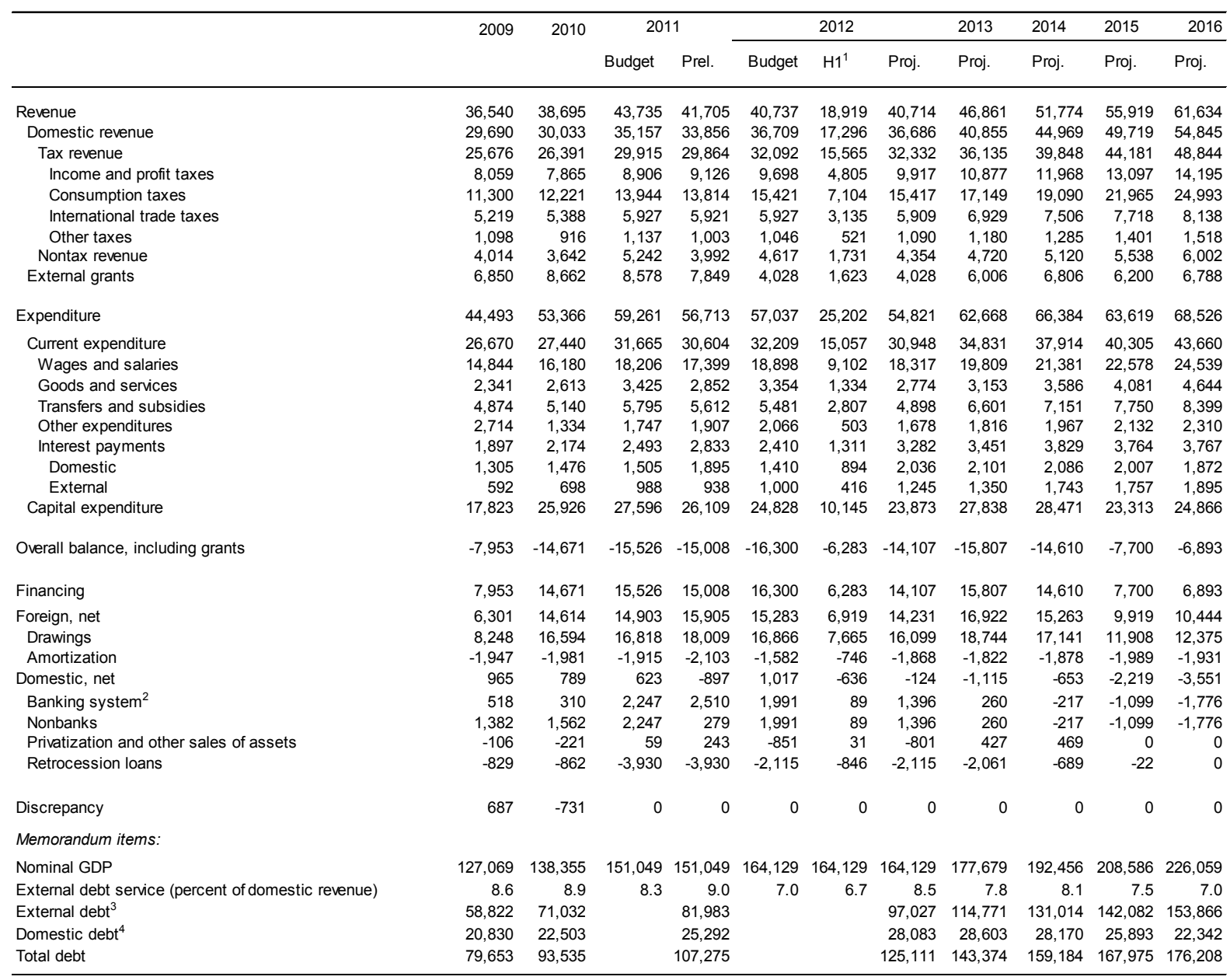

Sources: Ministry of Finance; Bank of Cape Verde; and IMF staff estimates and projections

\footnotetext{
${ }^{1}$ Authorities' fiscal plan.

${ }^{2}$ Includes adjustment for checks issued in late 2010, to pay for expenses appropriated in the 2010 budget, which were cashed in early 2011.

${ }^{3}$ Including external debt disbursements to the central government passed on to state-owned enterprises, which are responsible for servicing the foreign debt

The external debt stock corresponding to such disbursements is estimated at 5.5 billion escudos in 2011 .

${ }^{4}$ Net of central government deposits.
} 
Table 5b. Cape Verde: Fiscal Operations of the Central Government, 2009-16

(Percent of GDP)

\begin{tabular}{|c|c|c|c|c|c|c|c|c|c|c|c|}
\hline & \multirow[t]{2}{*}{2009} & \multirow[t]{2}{*}{2010} & \multicolumn{2}{|c|}{2011} & \multicolumn{3}{|c|}{2012} & \multirow{2}{*}{$\begin{array}{l}2013 \\
\text { Proj. }\end{array}$} & \multirow{2}{*}{$\begin{array}{l}2014 \\
\text { Proj. }\end{array}$} & \multirow{2}{*}{$\begin{array}{l}2015 \\
\text { Proj. }\end{array}$} & \multirow{2}{*}{$\begin{array}{l}2016 \\
\text { Proj. }\end{array}$} \\
\hline & & & Budget & Prel. & Budget & $\mathrm{H} 1^{1}$ & Proj. & & & & \\
\hline Revenue & 28.8 & 28.0 & 29.0 & 27.6 & 24.8 & 23.1 & 24.8 & 26.4 & 26.9 & 26.8 & 27.3 \\
\hline Domestic revenue & 23.4 & 21.7 & 23.3 & 22.4 & 22.4 & 21.1 & 22.4 & 23.0 & 23.4 & 23.8 & 24.3 \\
\hline Tax revenue & 20.2 & 19.1 & 19.8 & 19.8 & 19.6 & 19.0 & 19.7 & 20.3 & 20.7 & 21.2 & 21.6 \\
\hline Income and profit taxes & 6.3 & 5.7 & 5.9 & 6.0 & 5.9 & 5.9 & 6.0 & 6.1 & 6.2 & 6.3 & 6.3 \\
\hline Consumption taxes & 8.9 & 8.8 & 9.2 & 9.1 & 9.4 & 8.7 & 9.4 & 9.7 & 9.9 & 10.5 & 11.1 \\
\hline International trade taxes & 4.1 & 3.9 & 3.9 & 3.9 & 3.6 & 3.8 & 3.6 & 3.9 & 3.9 & 3.7 & 3.6 \\
\hline Other taxes & 0.9 & 0.7 & 0.8 & 0.7 & 0.6 & 0.6 & 0.7 & 0.7 & 0.7 & 0.7 & 0.7 \\
\hline Nontax revenue & 3.2 & 2.6 & 3.5 & 2.6 & 2.8 & 2.1 & 2.7 & 2.7 & 2.7 & 2.7 & 2.7 \\
\hline External grants & 5.4 & 6.3 & 5.7 & 5.2 & 2.5 & 2.0 & 2.5 & 3.4 & 3.5 & 3.0 & 3.0 \\
\hline Expenditure & 35.0 & 38.6 & 39.2 & 37.5 & 34.8 & 30.7 & 33.4 & 35.3 & 34.5 & 30.5 & 30.3 \\
\hline Current expenditure & 21.0 & 19.8 & 21.0 & 20.3 & 19.6 & 18.3 & 18.9 & 19.6 & 19.7 & 19.3 & 19.3 \\
\hline Wages and salaries & 11.7 & 11.7 & 12.1 & 11.5 & 11.5 & 11.1 & 11.2 & 11.1 & 11.1 & 10.8 & 10.9 \\
\hline Goods and services & 1.8 & 1.9 & 2.3 & 1.9 & 2.0 & 1.6 & 1.7 & 1.8 & 1.9 & 2.0 & 2.1 \\
\hline Transfers and subsidies & 3.8 & 3.7 & 3.8 & 3.7 & 3.3 & 3.4 & 3.0 & 3.7 & 3.7 & 3.7 & 3.7 \\
\hline Other expenditures & 2.1 & 1.0 & 1.2 & 1.3 & 1.3 & 0.6 & 1.0 & 1.0 & 1.0 & 1.0 & 1.0 \\
\hline Interest payments & 1.5 & 1.6 & 1.7 & 1.9 & 1.5 & 1.6 & 2.0 & 1.9 & 2.0 & 1.8 & 1.7 \\
\hline Domestic & 1.0 & 1.1 & 1.0 & 1.3 & 0.9 & 1.1 & 1.2 & 1.2 & 1.1 & 1.0 & 0.8 \\
\hline External & 0.5 & 0.5 & 0.7 & 0.6 & 0.6 & 0.5 & 0.8 & 0.8 & 0.9 & 0.8 & 0.8 \\
\hline Capital expenditure & 14.0 & 18.7 & 18.3 & 17.3 & 15.1 & 12.4 & 14.5 & 15.7 & 14.8 & 11.2 & 11.0 \\
\hline Overall balance, including grants & -6.3 & -10.6 & -10.3 & -9.9 & -9.9 & -7.7 & -8.6 & -8.9 & -7.6 & -3.7 & -3.0 \\
\hline Financing & 6.3 & 10.6 & 10.3 & 9.9 & 9.9 & 7.7 & 8.6 & 8.9 & 7.6 & 3.7 & 3.0 \\
\hline Foreign, net & 5.0 & 10.6 & 9.9 & 10.5 & 9.3 & 8.4 & 8.7 & 9.5 & 7.9 & 4.8 & 4.6 \\
\hline Drawings & 6.5 & 12.0 & 11.1 & 11.9 & 10.3 & 9.3 & 9.8 & 10.5 & 8.9 & 5.7 & 5.5 \\
\hline Amortization & -1.5 & -1.4 & -1.3 & -1.4 & -1.0 & -0.9 & -1.1 & -1.0 & -1.0 & -1.0 & -0.9 \\
\hline Domestic, net & 0.8 & 0.6 & 0.4 & -0.6 & 0.6 & -0.8 & -0.1 & -0.6 & -0.3 & -1.1 & -1.6 \\
\hline Banking system² & 0.4 & 0.2 & 1.5 & 1.7 & 1.2 & 0.1 & 0.9 & 0.1 & -0.1 & -0.5 & -0.8 \\
\hline Nonbanks & 1.1 & 1.1 & 1.5 & 0.2 & 1.2 & 0.1 & 0.9 & 0.1 & -0.1 & -0.5 & -0.8 \\
\hline Privatization and other sales of assets & -0.1 & -0.2 & 0.0 & 0.2 & -0.5 & 0.0 & -0.5 & 0.2 & 0.2 & 0.0 & 0.0 \\
\hline Retrocession loans & -0.7 & -0.6 & -2.6 & -2.6 & -1.3 & -1.0 & -1.3 & -1.2 & -0.4 & 0.0 & 0.0 \\
\hline Discrepancy & 0.5 & -0.5 & 0.0 & 0.0 & 0.0 & 0.0 & 0.0 & 0.0 & 0.0 & 0.0 & 0.0 \\
\hline \multicolumn{12}{|l|}{ Memorandum items: } \\
\hline Domestic debt ${ }^{3}$ & 16.4 & 16.3 & & 16.7 & & & 17.1 & 16.1 & 14.6 & 12.4 & 9.9 \\
\hline External debt ${ }^{4}$ & 46.3 & 51.3 & & 54.3 & & & 59.1 & 64.6 & 68.1 & 68.1 & 68.1 \\
\hline Total debt & 62.7 & 67.6 & & 71.0 & & & 76.2 & 80.7 & 82.7 & 80.5 & 77.9 \\
\hline
\end{tabular}

Sources: Ministry of Finance; Bank of Cape Verde; and IMF staff estimates and projections.

${ }^{1}$ Authorities' fiscal plan (using estimate of first-half GDP).

2 Includes adjustment for checks issued in late 2010, to pay for expenses appropriated in the 2010 budget, which were cashed in early 2011.

${ }^{3}$ Net of central government deposits.

${ }^{4}$ Including external debt disbursements to the central government passed on to state-owned enterprises, which are responsible

for servicing the foreign debt. The external debt stock corresponding to such disbursements is projected to be 3.6 percent of GDP in 2011 . 
Table 6. Cape Verde: Quantitative Assessment Criteria and Indicative Targets for 2010-11 Under the PSI ${ }^{1}$

\begin{tabular}{|c|c|c|c|c|c|c|c|c|c|c|c|c|}
\hline & \multicolumn{12}{|c|}{ Cumulative flows from end-Dec, 2010} \\
\hline & & & & & & & & & & & & \\
\hline & \multicolumn{3}{|c|}{ end-March } & \multicolumn{4}{|c|}{ end-June } & \multicolumn{4}{|c|}{ end-September } & \multirow{2}{*}{$\begin{array}{c}\text { end-December } \\
\text { Actual }\end{array}$} \\
\hline & $\begin{array}{c}\text { Assessment } \\
\text { criteria }\end{array}$ & $\begin{array}{c}\text { Adjusted } \\
\text { assessment } \\
\text { criteria }\end{array}$ & Actual & $\begin{array}{l}\text { Indicative } \\
\text { targets }\end{array}$ & $\begin{array}{c}\text { Adjusted } \\
\text { indicative } \\
\text { targets }\end{array}$ & Actual & Gap & $\begin{array}{l}\text { Assessment } \\
\text { criteria }\end{array}$ & $\begin{array}{c}\text { Adjusted } \\
\text { assessment } \\
\text { criteria }\end{array}$ & Actual & Gap & \\
\hline Quantitative targets & \multicolumn{11}{|c|}{ (Billions of Cape Verde escudos) } & \\
\hline Net domestic borrowing of the central government, ceiling ${ }^{2}$ & 0.9 & 2.0 & 1.1 & 1.5 & 3.8 & -0.1 & -3.8 & 2.9 & 5.4 & 1.7 & -3.7 & \\
\hline Net domestic assets of the central bank, ceiling ${ }^{2}$ & 0.3 & 2.4 & 3.2 & 0.6 & 1.6 & 6.0 & 4.5 & 0.8 & 3.6 & 6.6 & 3.0 & 1.8 \\
\hline \multirow[t]{2}{*}{ Nonaccumulation of new domestic arrears } & 0.0 & 0.0 & 0.0 & 0.0 & 0.0 & 0.0 & 0.0 & 0.0 & 0.0 & 0.0 & 0.0 & \\
\hline & \multicolumn{11}{|c|}{ (Millions of U.S. dollars) } & \\
\hline Nonaccumulation of new external arrears ${ }^{3}$ & 0.0 & 0.0 & 0.0 & 0.0 & 0.0 & 0.0 & 0.0 & 0.0 & 0.0 & 0.0 & 0.0 & \\
\hline $\begin{array}{l}\text { Contracting or guaranteeing nonconcessional external debt } \\
\text { with original maturity of more than one year, ceiling }{ }^{4}\end{array}$ & 35.0 & 35.0 & 0.0 & 35.0 & 35.0 & 0.0 & -35.0 & 35.0 & 35.0 & 0.0 & -35.0 & \\
\hline \multirow[t]{2}{*}{$\begin{array}{l}\text { Outstanding stock of nonconcessional external debt } \\
\text { with a maturity of less than one year, ceiling }{ }^{3,5}\end{array}$} & 0.0 & 0.0 & 0.0 & 0.0 & 0.0 & 0.0 & 0.0 & 0.0 & 0.0 & 0.0 & 0.0 & \\
\hline & \multicolumn{11}{|c|}{ (Millions of euros) } & \\
\hline Net international reserves, floor ${ }^{2}$ & 6.6 & -12.8 & -22.7 & 13.2 & 4.0 & -66.1 & -70.1 & 19.8 & -5.5 & -61.4 & -55.8 & -34.2 \\
\hline Indicative target & \multicolumn{11}{|c|}{ (Percent) } & \\
\hline Regulatory capital ratio of individual banks, floor $^{3}$ & 10.0 & 10.0 & 15.3 & 10.0 & 10.0 & 13.6 & & 10.0 & 10.0 & 14.5 & & \\
\hline \multicolumn{13}{|l|}{ Memorandum items: } \\
\hline Program assumptions & \multicolumn{11}{|c|}{ (Billions of Cape Verde escudos) } & \\
\hline $\begin{array}{l}\text { Nonproject external financial assistance, } \\
\text { including credit line (program assumption) }\end{array}$ & 1.2 & & 0.0 & 2.4 & & 0.2 & & 3.5 & & 1.4 & & \\
\hline External debt service & 0.9 & & 0.6 & 1.5 & & 1.3 & & 2.0 & & 2.1 & & \\
\hline Land sales & 0.2 & & 0.0 & 0.3 & & 0.0 & & 0.3 & & 0.0 & & \\
\hline External financial assistance, project and budget support loans & 5.9 & & 3.5 & 11.7 & & 10.5 & & 17.6 & & 14.8 & & \\
\hline
\end{tabular}

${ }^{1}$ Foreign currency amounts will be converted at current exchange rates.

${ }^{2}$ The ceiling or floor will be adjusted as specified in the TMU.

${ }^{3}$ Continuous.

${ }^{4}$ This assessment criterion applies not only to debt as defined in point No. 9 of the Guidelines on Performance Criteria with Respect to Foreign Debt (Decision No. 12274-(00/85), August 24, 2000, as amended on

August 31, 2009), but also commitments contracted or guaranteed for which value has not been received. Excluded from this performance criterion are debt rescheduling and debt reorganization, the Portuguese credit line, and borrowings from the IMF.

${ }^{5}$ The term "debt" has the meaning set forth in point No. 9 of the Guidelines on Performance Criteria with Respect to Foreign Debt (Decision No. 12274-(00/85), August 24, 2000, as amended on August 31, 2009).

Excluded from this performance criterion are rescheduling arrangements, the Portuguese credit line, borrowings from the IMF, and import-related financing. 
Table 7. Cape Verde: Structural Benchmarks for 2010-11

\begin{tabular}{|c|c|c|c|}
\hline $\begin{array}{l}\text { Objective/Macro } \\
\text { Criticality }\end{array}$ & Structural Benchmark & Timing & Status \\
\hline $\begin{array}{l}\text { Strengthen } \\
\text { securities market }\end{array}$ & $\begin{array}{l}\text { Issue fungible treasury bonds with common face and coupon values and } \\
\text { dates of payments. }\end{array}$ & end-June 2011 & Met \\
\hline $\begin{array}{l}\text { Strengthen tax } \\
\text { base }\end{array}$ & $\begin{array}{l}\text { Submit the draft individual and corporate income tax bills to the National } \\
\text { Assembly. }\end{array}$ & end-June 2011 & Not met \\
\hline $\begin{array}{l}\text { Promote fiscal } \\
\text { accountability }\end{array}$ & $\begin{array}{l}\text { Present cross debt between state-owned enterprises in the contingent } \\
\text { liabilities report and submit to the council of ministers. }\end{array}$ & end-June 2011 & Met \\
\hline $\begin{array}{l}\text { Strengthen the } \\
\text { financial sector }\end{array}$ & $\begin{array}{l}\text { The BCV and Ministry of Finance sign a memorandum of understanding to } \\
\text { expand and formalize the standing financial stability committee at the BCV. }\end{array}$ & end-September 2011 & Met with delay \\
\hline $\begin{array}{l}\text { Strengthen fiscal- } \\
\text { monetary policy } \\
\text { coordination }\end{array}$ & $\begin{array}{l}\text { The BCV and Ministry of Finance sign a fiscal-monetary coordination } \\
\text { agreement. }\end{array}$ & end-September 2011 & Met with delay \\
\hline $\begin{array}{l}\text { Strengthen debt } \\
\text { management }\end{array}$ & $\begin{array}{l}\text { Create a medium-term strategy report on the debt level, composition, and } \\
\text { desirable trend and present the report to the council of ministers. }\end{array}$ & end-September 2011 & Met \\
\hline $\begin{array}{l}\text { Strengthen the } \\
\text { financial sector }\end{array}$ & $\begin{array}{l}\text { Submit the financial framework law, which unifies the regulatory framework for } \\
\text { onshore and offshore banks, to the National Assembly. }\end{array}$ & end-December 2011 & Not met \\
\hline
\end{tabular}


Figure 1. Cape Verde: Monetary Developments

Net foreign assets declined significantly in the first half of 2011 but started to rebound in late $2011, \ldots$

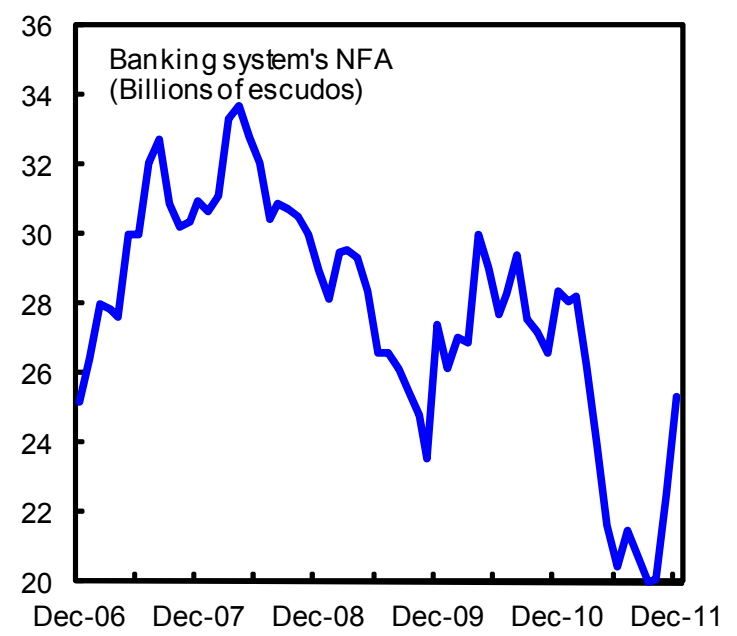

The growth of credit to the private sector stabilized in 2010 and first 10 months of 2011 but started to decline significantly in late 2011.

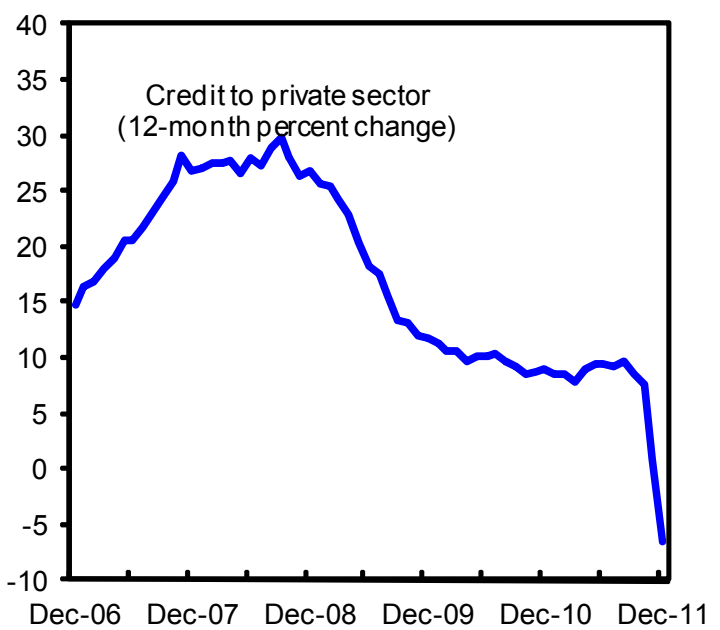

... While the central bank raised ts policy rate in January 2012 for the first time since 2009. Excess reserves have been declining. ${ }^{1}$

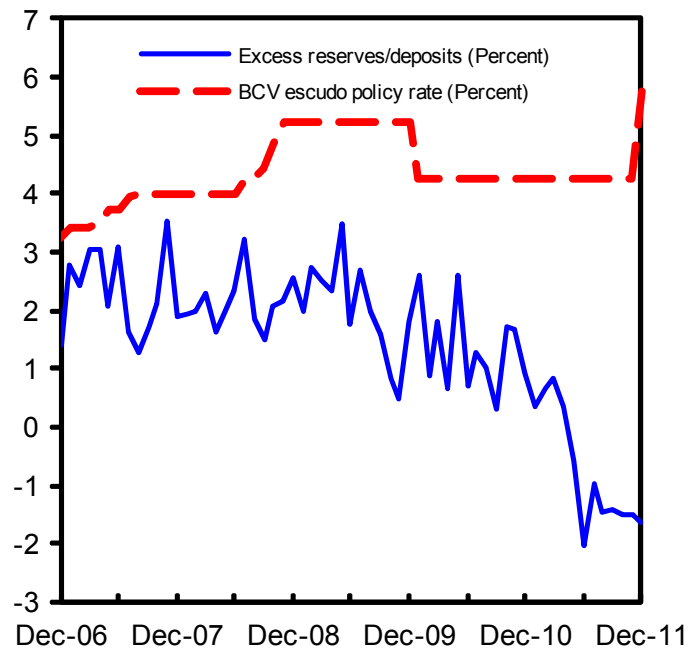

The growth rates of broad money and reserve money declined signifiicanlty in 2011.

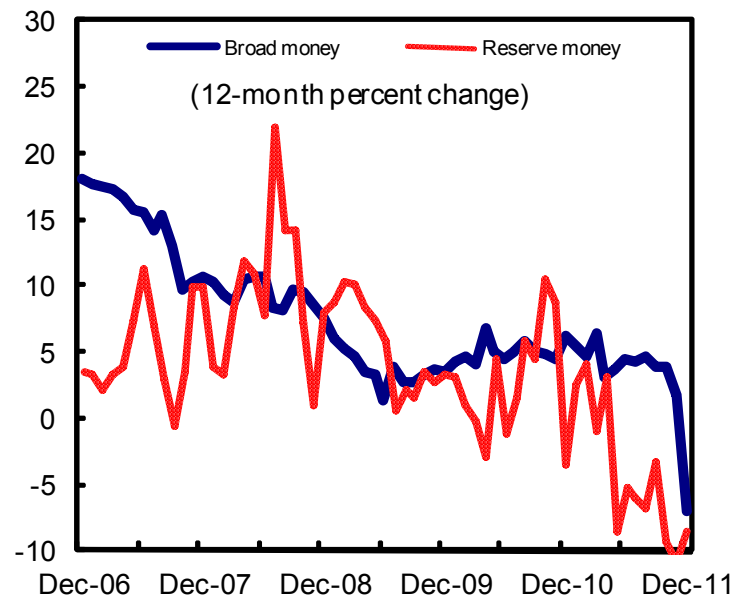

Source: Excess reserves are staff's calculations. All other data sources are from the Bank of Cape Verde.

${ }^{1}$ Excess reserves are a proxy for measuring, at the end of the month, the liquidity situation, and are defined as commercial banks' deposits at the central bank minus the total deposits of thecommercial banking system multiplied by the regulatory reserve requirement. A negative number does suggest a relative lack of market liquidity. However, the reserve requirement is calculated from average deposits over a certain period and bank reserves are measured at period end, so a negative number may also arise because of th is measurement anomaly. 
Figure 2. Cape Verde: External Sector Developments

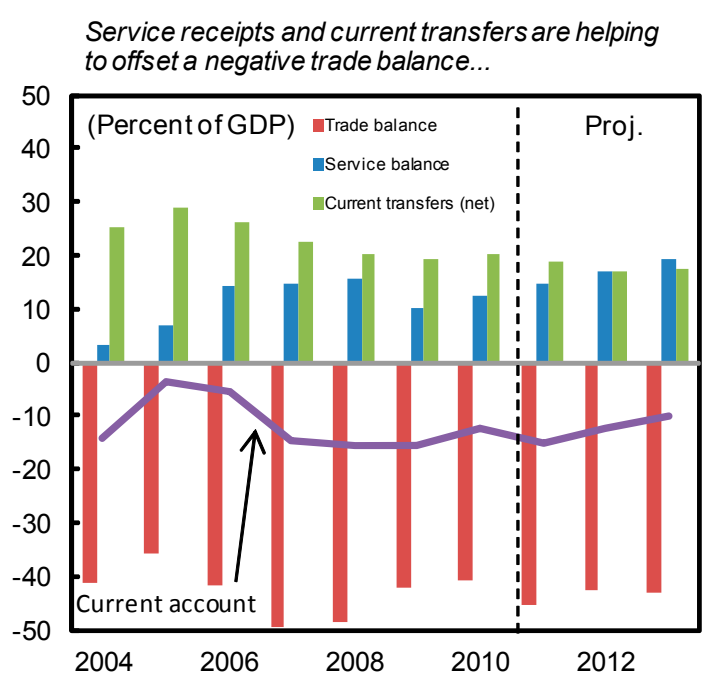

Concessional loans will continue to support the current account, but foreign direct investment has weakened in $2011 \ldots$

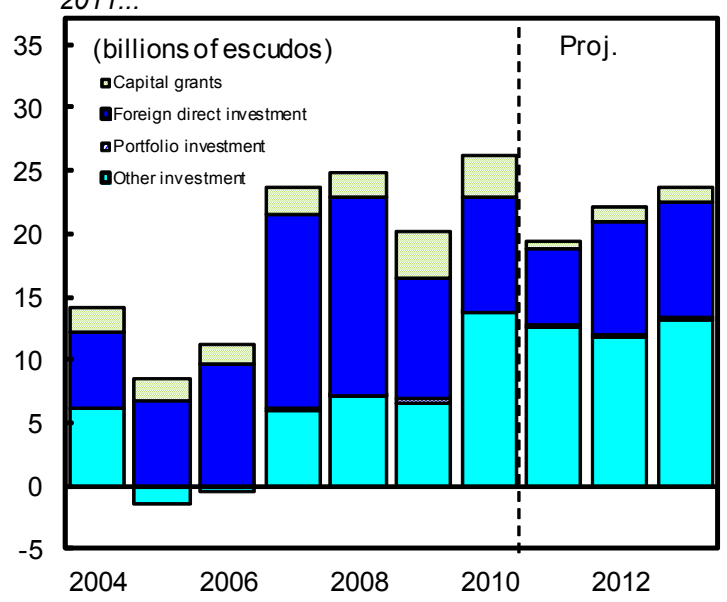

... and the trade deficit was mainly driven by goods imports.

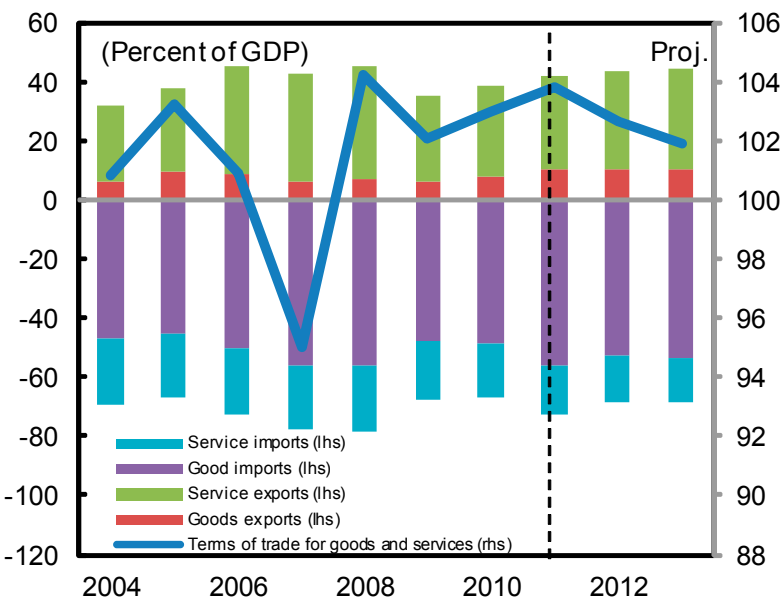

...and reserves show signs of weakening.

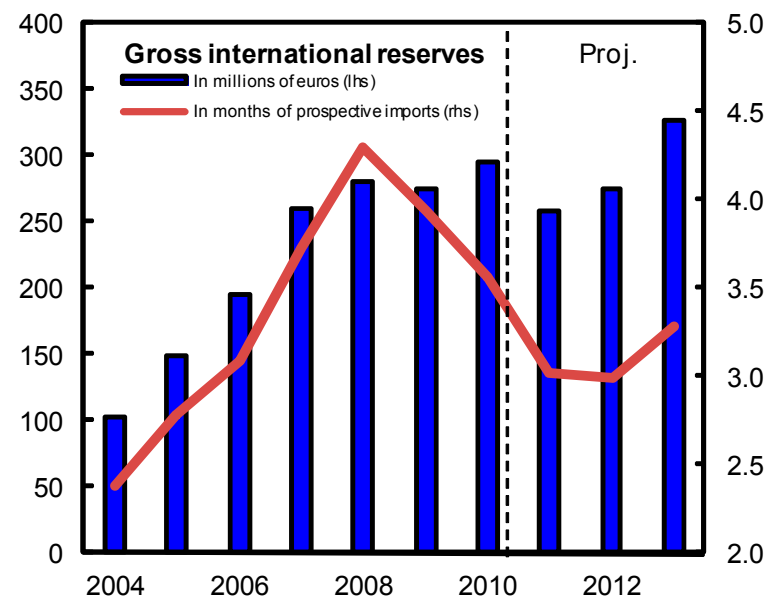

Sources: Cape Verdean auth orities and IMF staff estimates. 
Figure 3. Cape Verde: Fiscal Performance

(Percent of GDP)

The fiscal deficit began to decline in 2011 ...

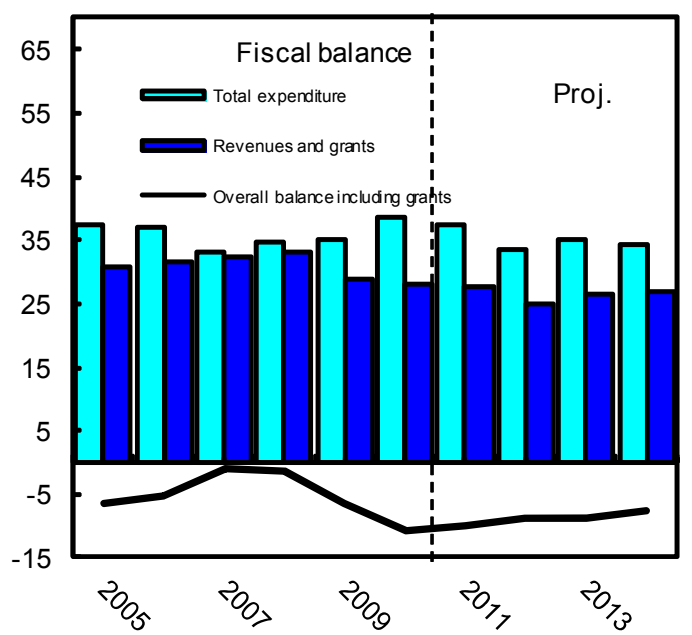

Tax revenues are stabilizing, broadly covering current expenditures.

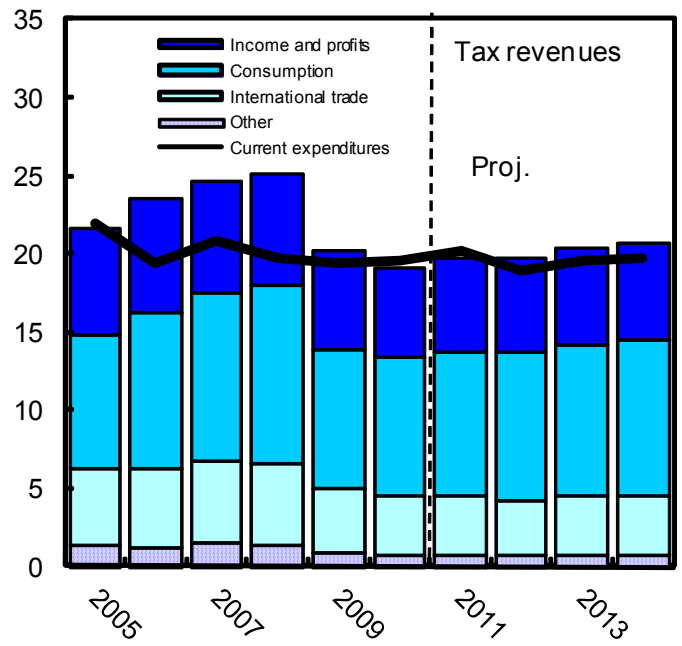

... as capital-spending began to decline.

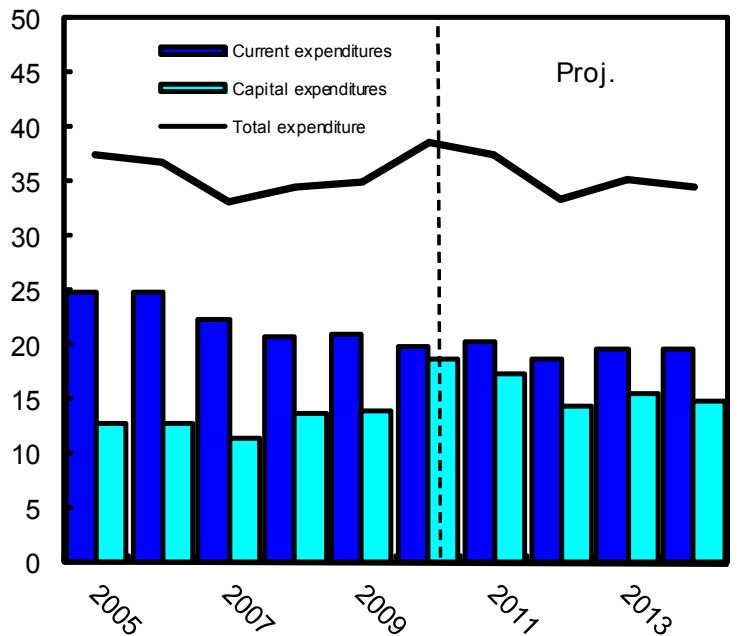

Domestic debt will continue to be roughly stable while external debt continues to increase.

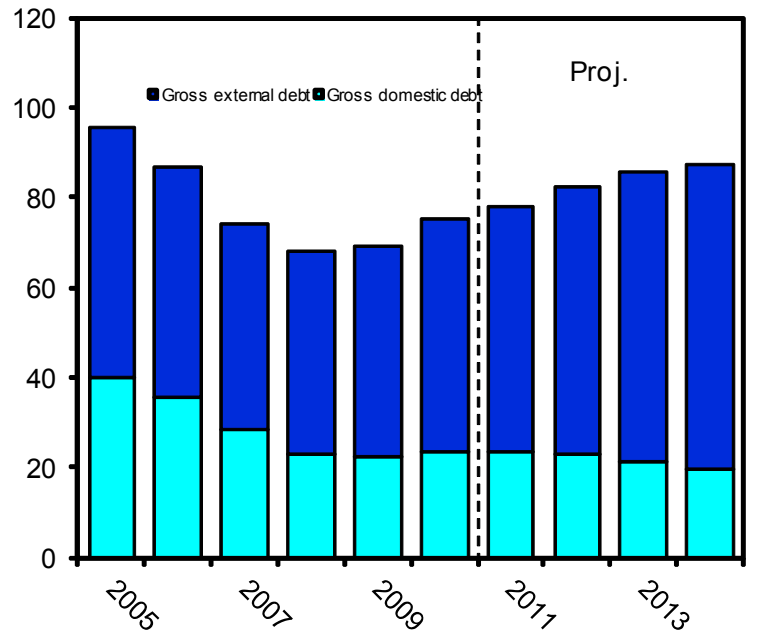

Sources: Cape Verdean authorities and IMF staff estimates. 


\section{APPENDIX I-LETTER OF INTENT}

January 5, 2012

Madame Christine Lagarde

Managing Director

International Monetary Fund

$70019^{\text {th }}$ Street, N.W.

Washington, D.C. 20431

USA

Dear Madame Lagarde:

1. Discussions for the concluding review of the Policy Support Instruments (PSI) were held in Praia during November 16-29, 2011. The PSI provided firm support for our policies for macroeconomic stability, economic growth, and poverty reduction.

2. We used the 15-month PSI program to support the government's policy goals. The government accelerated its investment program and increased social spending. Nonetheless, Cape Verde is confronting an adverse external environment and this has led to a sharply weaker balance of payments. The government cut spending to contain the budget deficit and support external current account adjustment. The BCV has tightened its monetary policy stance since mid 2011 to protect reserves. Inflation remains subdued.

3. All except two assessment criteria for end-September 2011 were met. The endSeptember NDA and NIR targets were missed by 2.0 percent of GDP and 4.1 percent of GDP, respectively, because of the expansion of credit to commercial banks and running down of government deposits, and the adverse external environment. We are taking corrective actions and are committed to bringing the monetary program back on track.

4. We met five benchmarks and made substantial progress on the remaining two structural benchmarks. The two outstanding benchmarks are: for submission of revised financial framework and tax laws to the parliament, which we expect to fulfill soon.

5. The economy continues to grow at a moderate rate and core inflation is contained, but the risks to our economic outlook are rising mainly because of the deteriorating external environment. Our short-term macroeconomic policy is committed to stabilize reserves at around 3 months of imports. Medium-term fiscal policy will aim to lower the deficit to safeguard debt sustainability and minimize domestic financing. Monetary policy will continue to be geared towards anchoring market expectations and safeguarding the peg. 
Further structural reforms are still needed to enhance debt management capacity, reform lossmaking state-owned enterprises, and strengthen financial sector reforms.

6. We request the completion of the second review under the PSI and waivers for the two missed end-September assessment criteria on the BCV's NDA and NIR. We authorize the IMF to publish this letter of intent, and the related staff report.

Sincerely yours,

$$
\text { /s/ }
$$

Cristina Duarte,

Minister of Finance

cc: Mr. Kossi Assimaidou, Executive Director

Mr. Carlos Burgo, Governor of Banco de Cabo Verde 
Table 1. Cape Verde: Quantitative Assessment Criteria and Indicative Targets for 2010-11 Under the PSI ${ }^{1}$

\begin{tabular}{|c|c|c|c|c|c|c|c|c|c|c|c|c|}
\hline & \multicolumn{12}{|c|}{ Cumulative flows from end-Dec, 2010} \\
\hline & \multicolumn{12}{|c|}{2011} \\
\hline & \multicolumn{3}{|c|}{ end-March } & \multicolumn{4}{|c|}{ end-June } & \multicolumn{4}{|c|}{ end-September } & \multirow{2}{*}{$\begin{array}{c}\text { end-December } \\
\text { Actual }\end{array}$} \\
\hline & $\begin{array}{l}\text { Assessment } \\
\text { criteria }\end{array}$ & $\begin{array}{c}\text { Adjusted } \\
\text { assessment } \\
\text { criteria } \\
\end{array}$ & Actual & $\begin{array}{l}\text { Indicative } \\
\text { targets }\end{array}$ & $\begin{array}{c}\text { Adjusted } \\
\text { indicative } \\
\text { targets } \\
\end{array}$ & Actual & Gap & $\begin{array}{l}\text { Assessment } \\
\text { criteria }\end{array}$ & $\begin{array}{c}\text { Adjusted } \\
\text { assessment } \\
\text { criteria } \\
\end{array}$ & Actual & Gap & \\
\hline Quantitative targets & \multicolumn{11}{|c|}{ (Billions of Cape Verde escudos) } & \multirow{6}{*}{1.8} \\
\hline Net domestic borrowing of the central government, ceiling ${ }^{2}$ & 0.9 & 2.0 & 1.1 & 1.5 & 3.8 & -0.1 & -3.8 & 2.9 & 5.4 & 1.7 & -3.7 & \\
\hline Net domestic assets of the central bank, ceiling ${ }^{2}$ & 0.3 & 2.4 & 3.2 & 0.6 & 1.6 & 6.0 & 4.5 & 0.8 & 3.6 & 6.6 & 3.0 & \\
\hline Nonaccumulation of new domestic arrears & 0.0 & 0.0 & 0.0 & 0.0 & 0.0 & 0.0 & 0.0 & 0.0 & 0.0 & 0.0 & 0.0 & \\
\hline \multicolumn{12}{|c|}{ (Millions of U.S. dollars) } & \\
\hline Nonaccumulation of new external arrears $^{3}$ & 0.0 & 0.0 & 0.0 & 0.0 & 0.0 & 0.0 & 0.0 & 0.0 & 0.0 & 0.0 & 0.0 & \\
\hline $\begin{array}{l}\text { Contracting or guaranteeing nonconcessional external debt } \\
\text { with original maturity of more than one year, ceiling }{ }^{4}\end{array}$ & 35.0 & 35.0 & 0.0 & 35.0 & 35.0 & 0.0 & -35.0 & 35.0 & 35.0 & 0.0 & -35.0 & \\
\hline $\begin{array}{l}\text { Outstanding stock of nonconcessional external debt } \\
\text { with a maturity of less than one year, ceiling } \\
\text { 3, } 5\end{array}$ & 0.0 & 0.0 & 0.0 & 0.0 & 0.0 & 0.0 & 0.0 & 0.0 & 0.0 & 0.0 & 0.0 & \\
\hline \multicolumn{13}{|c|}{ (Millions of euros) } \\
\hline Net international reserves, floor ${ }^{2}$ & 6.6 & -12.8 & -22.7 & 13.2 & 4.0 & -66.1 & -70.1 & 19.8 & -5.5 & -61.4 & -55.8 & -34.2 \\
\hline Indicative target & \multicolumn{11}{|c|}{ (Percent) } & \\
\hline Regulatory capital ratio of individual banks, floor ${ }^{3}$ & 10.0 & 10.0 & 15.3 & 10.0 & 10.0 & 13.6 & & 10.0 & 10.0 & 14.5 & & \\
\hline Memorandum items: & \multirow{2}{*}{\multicolumn{11}{|c|}{ (Billions of Cape Verde escudos) }} & \\
\hline Program assumptions & & & & & & & & & & & & \\
\hline $\begin{array}{l}\text { Nonproject external financial assistance, } \\
\text { including credit line (program assumption) }\end{array}$ & 1.2 & & 0.0 & 2.4 & & 0.2 & & 3.5 & & 1.4 & & \\
\hline External debt service & 0.9 & & 0.6 & 1.5 & & 1.3 & & 2.0 & & 2.1 & & \\
\hline Land sales & 0.2 & & 0.0 & 0.3 & & 0.0 & & 0.3 & & 0.0 & & \\
\hline External financial assistance, project and budget support loans & 5.9 & & 3.5 & 11.7 & & 10.5 & & 17.6 & & 14.8 & & \\
\hline
\end{tabular}

${ }^{1}$ Foreign currency amounts will be converted at current exchange rates.

${ }^{2}$ The ceiling or floor will be adjusted as specified in the TMU.

${ }^{3}$ Continuous.

${ }^{4}$ This assessment criterion applies not only to debt as defined in point No. 9 of the Guidelines on Performance Criteria with Respect to Foreign Debt (Decision No. 12274-(00/85), August 24, 2000, as amended on

August 31,2009 ), but also commitments contracted or guaranteed for which value has not been received. Excluded from this performance criterion are debt rescheduling and debt reorganization, the Portuguese credit

line, and borrowings from the IMF.

${ }^{5}$ The term "debt" has the meaning set forth in point No. 9 of the Guidelines on Performance Criteria with Respect to Foreign Debt (Decision No. 12274-(00/85), August 24, 2000, as amended on August 31, 2009).

Excluded from this performance criterion are rescheduling arrangements, the Portuguese credit line, borrowings from the IMF, and import-related financing.

CInternational Monetary Fund. Not for Redistribution 
Table 2. Cape Verde: Structural Benchmarks for 2010-11

\begin{tabular}{|c|c|c|c|}
\hline $\begin{array}{l}\text { Objective/Macro } \\
\text { Criticality }\end{array}$ & Structural Benchmark & Timing & Status \\
\hline $\begin{array}{l}\text { Strengthen } \\
\text { securities market }\end{array}$ & $\begin{array}{l}\text { Issue fungible treasury bonds with common face and coupon values and } \\
\text { dates of payments. }\end{array}$ & end-June 2011 & Met \\
\hline $\begin{array}{l}\text { Strengthen tax } \\
\text { base }\end{array}$ & $\begin{array}{l}\text { Submit the draft individual and corporate income tax bills to the National } \\
\text { Assembly. }\end{array}$ & end-June 2011 & Not met \\
\hline $\begin{array}{l}\text { Promote fiscal } \\
\text { accountability }\end{array}$ & $\begin{array}{l}\text { Present cross debt between state-owned enterprises in the contingent } \\
\text { liabilities report and submit to the council of ministers. }\end{array}$ & end-June 2011 & Met \\
\hline $\begin{array}{l}\text { Strengthen the } \\
\text { financial sector }\end{array}$ & $\begin{array}{l}\text { The BCV and Ministry of Finance sign a memorandum of understanding to } \\
\text { expand and formalize the standing financial stability committee at the BCV. }\end{array}$ & end-September 2011 & Met with delay \\
\hline $\begin{array}{l}\text { Strengthen fiscal- } \\
\text { monetary policy } \\
\text { coordination }\end{array}$ & $\begin{array}{l}\text { The BCV and Ministry of Finance sign a fiscal-monetary coordination } \\
\text { agreement. }\end{array}$ & end-September 2011 & Met with delay \\
\hline $\begin{array}{l}\text { Strengthen debt } \\
\text { management }\end{array}$ & $\begin{array}{l}\text { Create a medium-term strategy report on the debt level, composition, and } \\
\text { desirable trend and present the report to the council of ministers. }\end{array}$ & end-September 2011 & Met \\
\hline $\begin{array}{l}\text { Strengthen the } \\
\text { financial sector }\end{array}$ & $\begin{array}{l}\text { Submit the financial framework law, which unifies the regulatory framework for } \\
\text { onshore and offshore banks, to the National Assembly. }\end{array}$ & end-December 2011 & Not met \\
\hline
\end{tabular}




\section{APPENDiX II-CAPE VERDE: DebT SuStainabiLity ANALYSIS}

Cape Verde's debt remains sustainable, but risks have increased since the previous debt sustainability analysis (DSA) in November 2010. A faster accumulation of external public debt over the next few years combined with a deteriorating global economic environment accentuates macroeconomic vulnerabilities. Improvement of the debt outlook requires faster reduction of the fiscal deficit and implementation of long-standing structural reforms to boost growth. New public investment projects should directly support private sector investment and export growth.

\section{BACKGROUND}

1. This DSA updates an earlier DSA, covering 2010-30 (IMF Country Report No. 10/349). This earlier DSA found that public debt levels had increased as external borrowing was scaled up to finance public investment. The PV of external debt-to-GDP ratio was projected to approach the risk threshold in 2012 under the baseline, and to breach some stress tests. Debt service ratios remained below their thresholds, and resilient to the stress tests. The overall debt sustainability outlook was assessed as broadly unchanged compared to the previous DSA in 2008 (IMF Country Report No. 08/37).

2. This DSA reflects information available as of mid-October 2011 and uses the stock information at end-2010. The debt data currently covers central government external and domestic debt. External debt contracted by the central government on behalf of state-owned enterprises is also included. Publically-guaranteed debt extended to state-owned enterprises and local governments is included, but coverage is still hampered by data limitations. Technical assistance from the World Bank and the IMF during 2011 supported the authorities in improving measurement of the external debt stock and the associated projection of repayment flows. Both institutions assisted the authorities in the preparation of the Medium-Term Debt Strategy finalized in 2011.

\section{Public debt increased in $\mathbf{2 0 1 0}$ as the government implemented its public}

investment program. The nominal stock of public debt rose to 74.9 percent of GDP in 2010 from 68.8 percent in $2009^{1}$ The rise reflects a high level of public investment, which increased to 18.7 percent of GDP in 2010 from 14.0 percent in 2009. Total revenue worsened by around one percentage point of GDP during the period, but it was more than offset by a reduction in government current expenditures.

\footnotetext{
${ }^{1}$ The latest official nominal GDP estimates in Cape Verde date from 2007. The GDP estimates between 2008 and 2010 used in the DSA were constructed by the staff and are broadly aligned with the Ministry of Finance estimates. However, the BCV's GDP estimates are lower by about 10 percent for 2010, mainly reflecting differences in the deflator estimates.
} 
4. External public debt is mostly concessional. External public debt to GDP reached 51.3 percent in 2010 (Table 1). Multilateral institutions, and in particular, the World Bank Group and the African Development Bank, are the main external creditors (Text Figure). ${ }^{2}$ The portfolio has a long maturity profile and a low average interest rate, and over 95.0 percent is contracted at a fixed rate. Considering that the U.S. dollar corresponds to 41.9 percent of the SDR valuation basket, approximately 30.0 percent of the external debt stock is sensitive to the U.S. dollar-euro exchange rate fluctuations.

Text Figure. Cape Verde: External Indicators

External Debt by Creditor, 2010

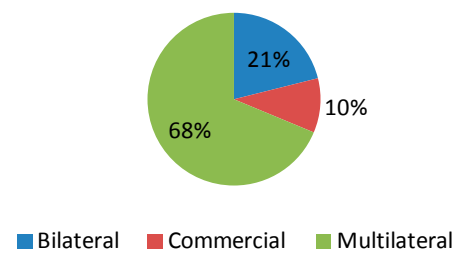

External Debt by Currency, 2010

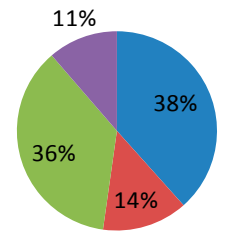

Euro $\square$ USD $\square$ SDR Other

Average Maturity and Interest Rate by External Creditor, 2010

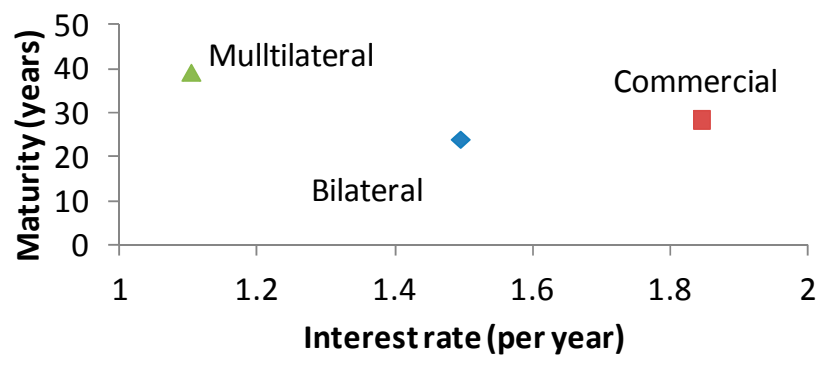

Source: Cape Verdean authorities.

${ }^{2}$ In December 2011, the African Development Bank disbursed a budget support loan in the amount of 15 million euros. This loan is on quasi-concessional basis and within the limit of non-concessional loans agreed in the context of the current PSI. 
5. External private debt poses a potential risk. The authorities are compiling information about private sector external debt, but there is a need for a more systematic monitoring of actual disbursements and repayment flows. Based on the available limited information, staff estimates that external private debt was 20 percent of GDP at the end of 2010-70 percent of this total corresponded to nonbank debt. The peg has facilitated this high level of debt, and the authorities need to factor this into their management of the exchange rate regime.

6. Domestic debt remains stable, at a manageable level. The authorities have been able to contain domestic financing of the central government. Between 2009 and 2010 , central government domestic debt remained at 23.5 percent of GDP. As of December 2009, the National Pension Fund (INPS) held approximately 35.0 percent of government domestic debt. The INPS has been running a surplus, on a current basis, and it is typically a key buyer of government debt in the primary market. The average interest rate on domestic debt is around 5.3 percent and average maturity is 7 years.

\section{From a consolidated perspective, staff estimates that the financial assets of the}

INPS appear to be larger than the liabilities of the state-owned enterprises and local governments. ${ }^{3}$ Guaranteed domestic debt of state-owned enterprises and municipalities amounted to an estimated 4.3 percent of GDP at end-2010. ${ }^{4}$ The INPS financial assets amounted to an estimated 18.1 percent of GDP at end-2009. Therefore, consolidation of the public sector seems to point to an overall lower level of debt than looking at the central government alone. ${ }^{5}$

\footnotetext{
${ }^{3}$ Government-guaranteed external debt is included in the central government's debt stock. There may be some additional unguaranteed external debt, which staff are working to ascertain.

${ }^{4}$ Approximately 75 percent of the domestic debt guarantees pertain to the loss-making electricity company (Electra).

${ }^{5}$ Data limitations preclude a thorough consolidation of public debt beyond the central government. Financial information on the loss-making airline company (TACV) and municipalities is limited. The government is working with the World Bank and independent auditors to determine the financial situation of TACV, and is carrying out a Public Expenditure Management and Financial Accountability (PEMFAR) exercise with support from donors to determine the financial situation of municipalities. Also, an actuarial analysis of the future liabilities of the INPS needs to be carried out.
} 


\section{The DSA BASELINE SCENARIO}

8. The DSA's macroeconomic assumptions reflect the negative spillovers from an uncertain external environment, and incorporate new medium-term fiscal projections. Cape Verde's near-term growth outlook has worsened, reflecting the protracted and complex nature of the economic adjustment that lies ahead in Europe (Text Table). As a result, near-term growth is projected to remain below the annual average of 6.2 percent observed between 2000 and 2010. The main transmission channels of external uncertainty are foreign direct investment inflows and weaknesses in the real estate sector. Against this backdrop, the government announced an acceleration of the public investment program, leading to a higher deficit trajectory in the near term. ${ }^{6}$

Text Table. Cape Verde: Growth and Fiscal Assumptions

\begin{tabular}{cccc}
\hline & $2011-15$ & $2016-31$ & $2011-31$ \\
\hline GDP growth (percent) & 5.2 & 5.5 & 5.5 \\
Updated DSA & 6.7 & 5.0 & 5.4 \\
Previous DSA & & & \\
Fiscal balance (percent of GDP) & -7.7 & -3.3 & -4.3 \\
$\quad$ Updated DSA & -5.6 & -2.5 & -3.3 \\
$\quad$ Previous DSA &
\end{tabular}

Sources: Cape Verdean authorities and IMF staff estimates.

* IMF Country Report No. 10/349.

\section{Long-term growth should benefit from gradual economic diversification.}

Physical capital accumulation from tourism- and emigrant-related investments has been a key driver of economic growth in Cape Verde. The government is committed to deepening economic diversification through development of fisheries, the seahub, and the financial and communication sectors. The baseline assumes diversification to bear fruit at a gradual pace, and the annual average growth rate will remain below its historic average over the past decade. ${ }^{7}$ This is in line with international experiences whereby economic diversification of small island states constitutes a significant development challenge.

\section{The largest projects of the public investment program are in infrastructure.}

Almost half of the entire public capital spending is in the energy, water and transportation sectors. Deficiencies in infrastructure in these sectors constitute clear bottlenecks for private sector development. The public investment program also includes a social component,

\footnotetext{
${ }^{6}$ The public investment acceleration is being financed on concessional terms. Upon graduation from the least developed country (LDC) category in 2007, the international donor community agreed to provide a five-year window in which Cape Verde could still access concessional loans. Negotiations are on-going to extend the transition period until 2015. Cape Verde needs to reconsider its overall public investment program and the need now to build up fiscal buffers at a quicker pace.

${ }^{7}$ Staff is taking a relatively modest view about the growth dividends from the large infrastructure investment program, in light of the weak external environment.
} 
human capital development, and sector-specific interventions to improve competitiveness (mainly in agriculture and fisheries).

\section{The baseline scenario is conditional on the government's commitment to low} domestic financing. The authorities have successfully controlled current expenditure to accommodate revenue shortfalls and public investment increases so far. Going forward, it is assumed that the government will continue to control fast-growing components of current expenditures, and to make progress on the implementation of long-standing tax policy reforms. Increasing domestic revenue as a percent of GDP by broadening the tax base and rationalizing fiscal exemptions is critical to avoid excessive expenditure compression. ${ }^{8}$ Finally, the government is assumed to finance investment with relatively little recourse to domestic sources to support the exchange rate regime.

\section{The baseline scenario assumes that monetary policy will react promptly to}

stabilize reserves. The central bank is assumed to tighten and rein in domestic credit growth to avoid reserve losses when necessary. Despite an increased vulnerability of the external accounts, ${ }^{9}$ reserve coverage remains at a minimally satisfactory level and the baseline scenario assumes the peg is sustainable.

\section{EXTERnal DebT Sustainability}

\section{Baseline scenario}

13. The baseline scenario points to a deterioration of the external debt outlook in the next five years, followed by a gradual improvement. The debt sustainability analysis uses policy-dependent external debt thresholds to gauge risks. Cape Verde is considered to have a strong policy performance, based on the latest World Bank's Country Policy and Institutional Assessment, thereby giving it slightly higher thresholds than countries with weaker policies. There is no significant or protracted breach of external debt indicators through 2031, but the present value (PV) of the debt-to-GDP approaches its policy-dependent threshold in 2016. The PV of debt-to-exports and the PV of debt-to-revenue remain well below their respective thresholds, reflecting the concessionality of outstanding debt and new external borrowing in the near-term. ${ }^{10}$ The main message from the baseline scenario is broadly similar to the one from the previous DSA, despite differences in the debt profiles which are mostly explained by

\footnotetext{
${ }^{8}$ The World Bank and the IMF are working with the authorities on tax policy reform.

${ }^{9}$ International reserve coverage is projected to average 3.0 months of prospective imports between 2011 and 2015 , against an average of 4.1 months projected in the previous DSA for the same period.

${ }^{10}$ Cape Verde lost a substantial amount of international reserves during 2011, which explains the large residual in Table 1. The authorities took corrective policy actions to tighten domestic macroeconomic policies, and counter the impact of the negative external environment.
} 
an acceleration of the public investment program and improved measurement of the external debt. ${ }^{11}$

\section{Box 1. Macroeconomic Assumptions for the Baseline Scenario}

Real GDP growth experienced a solid recovery from the 2008 global crisis, remaining resilient until the first half of 2011, when growth started to slow down reflecting the troubled external environment. Hence, the medium-term outlook is weaker. Cape Verde's long-term growth prospects continue to be favorable, underpinned by a dynamic tourism sector and infrastructure investment.

Inflation is assumed to remain anchored to international levels given the peg to the euro, but also influenced by external volatility. Wage moderation and price flexibility, particularly in the tourism sector, will continue to facilitate adjustment to shocks.

External balance is restored. The current account is projected to remain negative during the DSA horizon due to the country's structural dependence on imports, but improve over time as exports become more diversified and import shares decline. Current transfers as a percent of GDP are expected to slowly decline as official grants are phased out and the domestic economy gradually outgrows remittance inflows. Capital account flows are expected to be modestly lower, particularly as the temporary increase in official disbursements to finance the public investment program comes to an end.

The overall fiscal balance is projected to improve, but remains a concern. After peaking in 2010 at doubledigits, the fiscal deficit as a percentage of GDP is projected to decline and settle at an annual average of 3.3 percent in 2016-31. Tax revenue is expected to increase by about 4 percentage points of GDP over the DSA horizon due to the implementation of tax reforms and to the broadening of the tax base. External grants will decline over time, broadly offsetting tax revenue gains. Current expenditure is expected to grow in line with nominal GDP, while capital expenditure should reduce from its current high levels (18.7 percent of GDP in 2010) to less than 10.0 percent of GDP in the outer years.

Financing: The concessionality of new external loans will decline significantly in the medium term. The baseline assumes that this will happen around 2018-20 when concessional loans contracted up until 2013-15 should be close to full disbursement. Additionally, the baseline assumes that the authorities will continue to keep domestic borrowing low to support the peg.

\section{Alternative scenarios and stress tests}

\section{Bound tests and alternative scenarios show that Cape Verde's external debt is vulnerable to large shocks and a deteriorated economic environment (Table 2 and} Figure 1). The PV of external debt-to-GDP breaches the relevant thresholds under different stress tests. The exchange rate shock causes the largest and most protracted breach, highlighting the criticality of safeguarding the peg. ${ }^{12}$ The export shock and the U.S. dollar GDP deflator shock also lead to breaches, though much less pronounced. ${ }^{13}$ Additionally,

\footnotetext{
${ }^{11}$ In some cases, future disbursements of contracted debt were included as outstanding debt and/or had their repayment flows noted as service payments of the existing debt stock. Such disbursements were also recorded as external financing in the Medium-Term Fiscal Framework, leading to an overestimation of the debt stock and present value measures. The World Bank and IMF staff worked with the authorities during 2011 to assure consistency of public external debt data used in this DSA.

${ }^{12}$ A one-time 30 percent nominal depreciation relative to the baseline in 2012 is assumed.

${ }^{13}$ Export value growth at historical average minus one standard deviation in 2012-13; U.S. dollar GDP deflator at historical average minus one standard deviation in 2012-13.
} 
under the assumption that new public sector loans are made on less favorable terms, the PV of external debt-to-GDP suffers a protracted breach of the relevant threshold. ${ }^{14}$ Stress tests and less favorable assumptions provide some comfort about the repayment capacity of Cape Verde, as they do not imply any breach of the relevant thresholds for the PV of debt-toexports and the PV of debt-to-revenue.

\section{A tail risk scenario resulting from severe disruption in the external environment} could put significant pressure on debt indicators. In the event of a strong deterioration of external conditions, Cape Verde could face tail risks involving a combination of negative shocks. Staff simulated the impact of an alternative scenario in which: (i) growth and foreign direct investment fall by 50.0 percent from their baseline projections for each year from 2012 to 2014; (ii) exports as a percentage of GDP fall by 10.0 percent from its baseline projection for each year from 2012 to 2014; and (iii) the interest rate on the marginal external debt needed to finance the balance of payments reaches five percent with a grace period of two years. The results indicate that the margin of comfort for some of the debt indicators under the baseline can be quickly eroded in this situation. Private sector external debt represents another source of vulnerability in such an extreme scenario, given potential higher interest costs associated with a higher country risk premium.

\section{Public Sector Debt Sustainability}

\section{Baseline scenario}

\section{Total public debt in the baseline scenario reaches high levels and mirrors} external debt dynamics. Domestic debt is projected to remain manageable and to decline gradually over the medium term to support the exchange rate regime. However, public debt to GDP rises to high levels owing to large foreign-financed fiscal deficits, peaking at 87.4 percent in 2014 (Table 3). For the longer term, the domestic debt is projected to pick up slightly, remaining below 20.0 percent of GDP, as reliance on external financing declines more significantly.

\section{Alternative scenarios and stress tests}

17. The debt outlook is vulnerable to the realization of losses on contingent liabilities associated with state-owned enterprises. The latest government report on liabilities of selected state-owned enterprises covers six public companies. ${ }^{15}$ The report indicates that underlying contingent liabilities associated to guaranteed and non-guaranteed debt reached 11.5 percent of GDP in 2010. Updated financial information on the debt of the loss-making

\footnotetext{
${ }^{14}$ This is an important alternative scenario to bear in mind given the existing concessionality of Portuguese credit lines, which rely on subsidies from that country's treasury.

${ }^{15}$ The Cape Verdean government holds a controlling stake in 13 financial and non-financial companies (more than 50.0 percent). The report covers key state-owned enterprises, but improving coverage is important to provide a more comprehensive assessment of risks.
} 
TACV is lacking, which limits analysis. In any case, it is clear that both TACV and Electra represent a high fiscal risk to the budget. Both companies regularly need to access short-term credit facilities and resort to government guarantees. The government is committed to addressing the issues and the international community is providing support for restructuring. ${ }^{16}$

\section{Public debt sustainability indicators indicate vulnerability to alternative} scenarios and stress tests (Table 4 and Figure 2). However, the alternative scenario of an unchanged primary balance from 2011 , which leads to a clearly unsustainable debt dynamics, is not a likely scenario since the scale-up of capital spending is expected to be temporary. Staff has also simulated the impact of a scenario of fiscal deterioration in which (i) domestic revenue as a percent of GDP remain stagnant at the 2011 level and (ii) current expenditure as a percent of GDP grows five percent faster than in the baseline projection for every year. The results indicate the critical importance of implementing the long-standing tax policy reforms and controlling growth of current expenditures.

\section{Conclusions}

19. Staff assesses Cape Verde's debt as sustainable. The debt indicators are projected to improve as the public investment program winds down. The long maturities and high concessionality of the existing debt mitigate rollover risks for now. Domestic revenue covers current expenditure, providing some room for adjustment in case there is an unexpected reduction of official grants or foreign financing. Cape Verde enjoys strong credibility due to its policies and democratic institutions, and continues to galvanize support from the international community. The tourism sector has remained resilient and competitive. International reserves are at minimally adequate levels and the authorities are committed to protecting the peg.

\section{The faster accumulation of external public debt in the midst of an uncertain} global environment has increased risks around the baseline. The prospects for the world economy have deteriorated over the last year. While Cape Verde has withstood well these tough times, staff believes that it is crucial to bolster progress on macroeconomic stability achieved so far. To that end, the authorities should seek faster debt reduction. This should involve moderation of the public investment pace, particularly domestically financed investment. Postponing new foreign-financed investments, especially those assessed to have more indefinite returns to the private sector, would also be prudent. ${ }^{17}$ Such a strategy would also help to promote external balance and shore up international reserves by containing

\footnotetext{
${ }^{16}$ The World Bank expects to approve in 2012 a large energy loan to Electra (US\$60 million) which will address priority investments and operational losses, and support the company's reform. The World Bank is also engaged with TACV.

${ }^{17}$ Public investment in Cape Verde as a percent of GDP (18.7 percent in 2010) was more than twice the sub-Saharan average (7.4 percent) and the highest among middle-income African economies (Botswana, Cape Verde, Ghana, Lesotho, Mauritius, Namibia, Senegal, Seychelles, South Africa, Swaziland, and Zambia).
} 
growth of the import bill during uncertain times. Regarding debt management, the authorities should prioritize the development of analytical capacity in the Ministry of Finance to inform policymakers of risks around the baseline.

\section{The authorities view the rapid build-up of public debt as necessary to promote} the country's development as a middle-income economy. A key government objective is to create the foundations for economic diversification through public infrastructure investment. In their view, the temporary availability of concessional funds is a unique opportunity that cannot be missed. Staff understands the government's rationale, but it is of the view that public investment should be predicated on more conservative assumptions about the benefits of this investment, and thus undertaken more cautiously to match new external risk conditions and actual progress on structural reforms. ${ }^{18}$ The staff's views and underlying assumptions of this DSA were discussed with the authorities.

\footnotetext{
${ }^{18}$ Slow progress on structural reforms has been holding back competitiveness for some time. There is general agreement about which reforms are needed: tax policy, revenue administration, state-owned enterprise restructuring (particularly of Electra and TACV), labor market flexibility, public investment management, and financial sector legislation. However, despite the commitment of the authorities, broader political traction has been lacking at times.
} 
Table 1.Cape Verde: External Debt Sustainability Framework, Base line Scenario, 2008-2031 1/

(In percent of GDP, unless otherwise indicated)

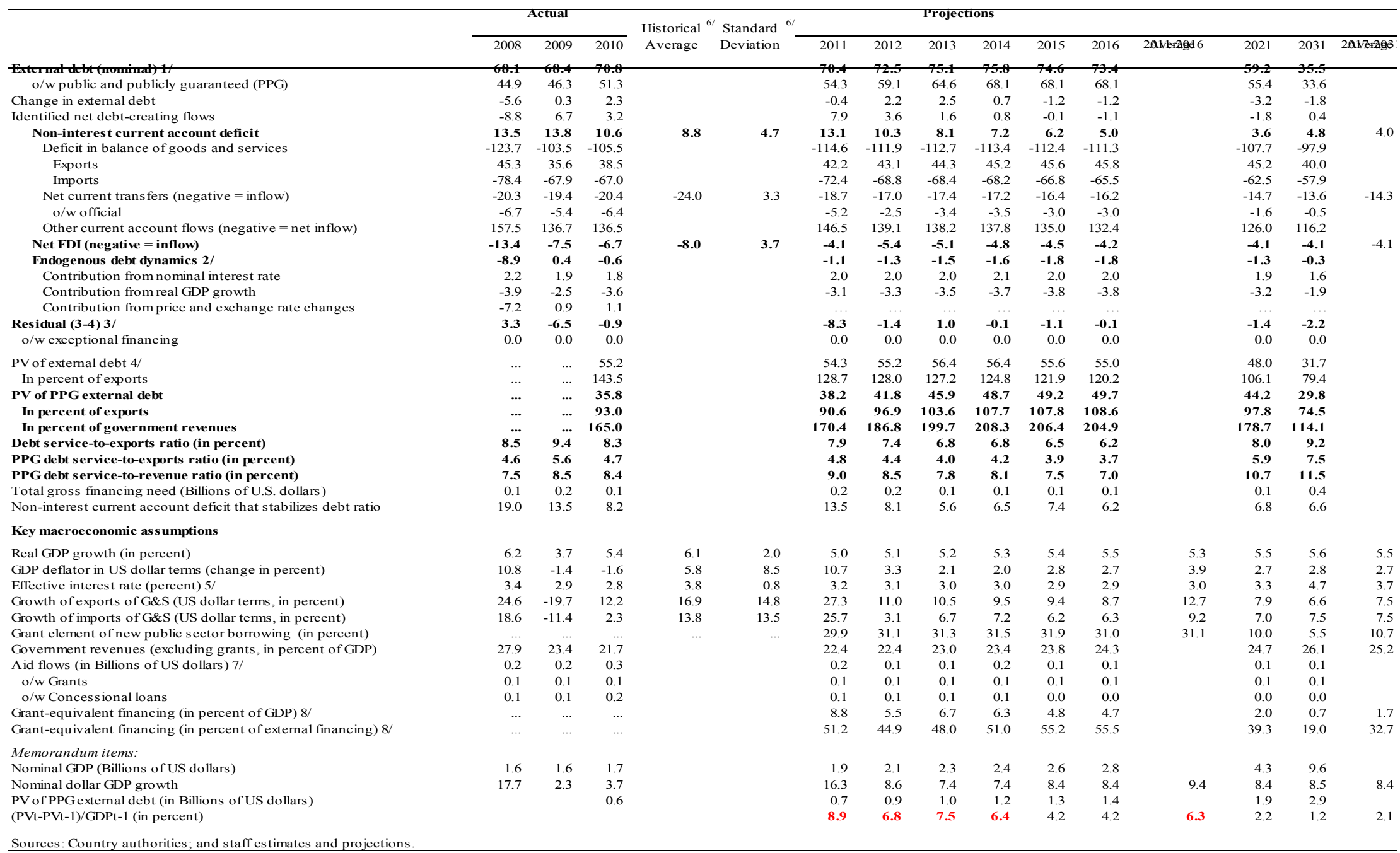

Sources: Country authorities; and staff estimates and $\mathrm{p}$

2/ Derived as $[r-g-\rho(1+g)] /(1+g+\rho+g \rho)$ times previous period debt ratio, with $r=$ nominal interest rate; $g=$ real GDP growth rate, and $\rho=$ growth rate of GDP deflator in U.S. dollar terms.

3 Includes exceptional financing (i.e., changes in arrears and debt relief); changes in gross foreign assets; and valuation adjustments. For projections also includes contribution from price and exchange rate changes.

4/ Assumes that PV of private sector debt is equivalent to its face value.

$5 /$ Current-year interest payments divided by previous period debt stock.

6/ Historical averages and standard deviations are generally derived over the past 10 years, subject to data availability.

7/ Defined as grants, concessional loans, and debt relief.

8/ Grant-equivalent financing includes grants provided directly to the government and through new borrowing (difference between the face value and the PV of new debt).

CInternational Monetary Fund. Not for Redistribution 
Table 2.Cape Verde: Sensitivity Analysis for Key Indicators of Public and Publicly Guaranteed External Debt, 2011-2031 (In percent)

\begin{tabular}{llllllll}
\hline 2011 & 2012 & 2013 & 2014 & 2015 & 2016 & 2021 & 2031
\end{tabular}

PV of debt-to GDP ratio

\section{Baseline}

A. Atternative Scenarios

A1. Key variables at their historical averages in 2011-2031 1/

A2. New public sector loans on less favorable terms in 2011-2031 2

A3. Alternative Scenario : External tail risk

$38 \quad 42$

\section{B. Bound Tests}

B1. Real GDP growth at historical average minus one standard deviation in 2012-2013

B2. Export value growth at historical average minus one standard deviation in 2012-2013 3/

B3. US dollar GDP deflator at historical average minus one standard deviation in 2012-2013

B4. Net non-debt creating flows at historical average minus one standard deviation in 2012-2013 4/

B5. Combination of B1-B4 using one-half standard deviation shocks

B6. One-time 30 percent nominal depreciation relative to the baseline in 20125 /

PV of debt-to-exports ratio

\section{Baseline}

\section{A. Alternative Scenarios}

A1. Key variables at their historical averages in 2011-2031 1/

A2. New public sector loans on less favorable terms in 2011-2031 2

A3. Alternative Scenario : External tail risk

\section{B. Bound Tests}

B1. Real GDP growth at historical average minus one standard deviation in 2012-2013

B2. Export value growth at historical average minus one standard deviation in 2012-2013 3/

B3. US dollar GDP deflator at historical average minus one standard deviation in 2012-2013

B4. Net non-debt creating flows at historical average minus one standard deviation in 2012-2013 4/

B5. Combination of B1-B4 using one-half standard deviation shocks

B6. One-time 30 percent nominal depreciation relative to the baseline in 20125 /

$\begin{array}{llllllll}38 & 36 & 37 & 37 & 37 & 37 & \mathbf{3 4} & 16 \\ 38 & 44 & 51 & 56 & 57 & 58 & \mathbf{5 5} & 43 \\ 38 & 50 & 62 & 66 & 60 & 53 & \mathbf{4 8} & 32 \\ & & & & & & & \\ 38 & 42 & 47 & 49 & 50 & 50 & \mathbf{4 5} & 30 \\ 38 & 45 & 56 & 57 & 56 & 54 & \mathbf{4 4} & 30 \\ 38 & 44 & 51 & 54 & 54 & 54 & \mathbf{4 9} & 33 \\ 38 & 39 & 41 & 44 & 45 & 46 & \mathbf{4 4} & 30 \\ 38 & 37 & 37 & 41 & 43 & 45 & \mathbf{4 5} & 31 \\ 38 & 59 & 64 & 68 & 69 & 69 & \mathbf{6 2} & 42\end{array}$

\section{PV of debt-to-revenue ratio}

Baseline

\section{A. Alternative Scenarios}

A1. Key variables at their historical averages in 2011-2031 1/

A2. New public sector loans on less favorable terms in 2011-2031 2

A3. Alternative Scenario : External tail risk

\section{B. Bound Tests}

B1. Real GDP growth at historical average minus one standard deviation in 2012-2013 B2. Export value growth at historical average minus one standard deviation in 2012-2013 3/

B3. US dollar GDP deflator at historical average minus one standard deviation in 2012-2013

B4. Net non-debt creating flows at historical average minus one standard deviation in 2012-2013 4/

B5. Combination of B1-B4 using one-half standard deviation shocks

B6. One-time 30 percent nominal depreciation relative to the baseline in $20125 /$

91

97

104

108

108

$\begin{array}{rrrrrrrr}91 & 97 & 103 & 107 & 107 & 106 & \mathbf{9 8} & 75 \\ 91 & 113 & 147 & 149 & 143 & 138 & \mathbf{1 1 5} & 87 \\ 91 & 97 & 103 & 107 & 107 & 106 & \mathbf{9 8} & 75 \\ 91 & 90 & 92 & 98 & 99 & 101 & \mathbf{9 8} & 75 \\ 91 & 86 & 84 & 91 & 95 & 99 & \mathbf{9 9} & 76 \\ 91 & 97 & 103 & 107 & 107 & 106 & \mathbf{9 8} & 75\end{array}$

$\begin{array}{llllllll}170 & 188 & 203 & 212 & 208 & 205 & \mathbf{1 8 2} & 116 \\ 170 & 201 & 242 & 246 & 233 & 221 & \mathbf{1 7 9} & 114 \\ 170 & 198 & 222 & 231 & 227 & 224 & \mathbf{1 9 9} & 127 \\ 170 & 175 & 177 & 189 & 190 & 191 & \mathbf{1 7 8} & 114 \\ 170 & 166 & 162 & 177 & 182 & 187 & \mathbf{1 8 2} & 117 \\ 170 & 262 & 280 & 293 & 287 & 283 & \mathbf{2 5 2} & 161\end{array}$

\section{CInternational Monetary Fund. Not for Redistribution}


Table 2.Cape Verde: Sensitivity Analysis for Key Indicators of Public and Publicly Guaranteed External Debt, 2011-2031 (continued) (In percent)

\section{Debt service-to-exports ratio}

\section{Baseline}

A Alternative Scenarios

A1. Key variables at their historical averages in 2011-2031 1/

A2. New public sector loans on less favorable terms in 2011-2031

A3. Alternative Scenario : External tail risk

\section{B. Bound Tests}

B1. Real GDP growth at historical average minus one standard deviation in 2012-2013

B2. Export value growth at historical average minus one standard deviation in 2012-2013

B3. US dollar GDP deflator at historical average minus one standard deviation in 2012-2013

B4. Net non-debt creating flows at historical average minus one standard deviation in 2012-2013 4/

B5. Combination of B1-B4 using one-half standard deviation shocks

B6. One-time 30 percent nominal depreciation relative to the baseline in 20125

Debt service-to-revenue ratio

\section{Baseline}

\section{A. Alternative Scenarios}

A1. Key variables at their historical averages in 2011-2031 1/

A2. New public sector loans on less favorable terms in 2011-2031

A3. Alternative Scenario : External tail risk

\section{B. Bound Tests}

B1. Real GDP growth at historical average minus one standard deviation in 2012-2013

B2. Export value growth at historical average minus one standard deviation in 2012-2013 3/

B3. US dollar GDP deflator at historical average minus one standard deviation in 2012-2013

B4. Net non-debt creating flows at historical average minus one standard deviation in 2012-2013 4/

B5. Combination of B1-B4 using one-half standard deviation shocks

B6. One-time 30 percent nominal depreciation relative to the baseline in 20125

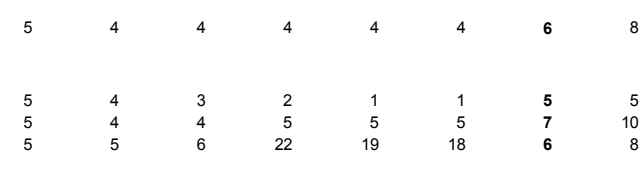

Memorandum item:

Grant element assumed on residual financing (i.e., financing required above baseline) 6

$\begin{array}{rrrrrrrr}5 & 4 & 4 & 4 & 4 & 4 & 6 & 8 \\ 5 & 5 & 5 & 7 & 10 & 9 & 7 & 9 \\ 5 & 4 & 4 & 4 & 4 & 4 & 6 & 8 \\ 5 & 4 & 4 & 3 & 2 & 2 & 6 & 8 \\ 5 & 4 & 4 & 1 & 0 & 0 & 6 & 8 \\ 5 & 4 & 4 & 4 & 4 & 4 & 6 & 8\end{array}$

Sources: Country authorities; and staff estimates and projections.

1/ Variables include real GDP growth, growth of GDP deflator (in U.S. dollar terms), non-interest current account in percent of GDP, and non-debt creating flows.

2/ Assumes that the interest rate on new borrowing is by 2 percentage points higher than in the baseline., while grace and maturity periods are the same as in the baseline.

3 / Exports values are assumed to remain permanently at the lower level, but the current account as a share of GDP is assumed to return to its baseline level after the shock (implicitly assuming

an offsetting adjustment in import levels).

I Includes official and private transfers and FDI.

$5 /$ Depreciation is defined as percentage decline in dollar/local currency rate, such that it never exceeds 100 percent

$6 /$ Applies to all stress scenarios except for A2 (less favorable financing) in which the terms on all new financing are as specified in footnote 2. 
Table 3.Cape Verde: Public Sector Debt Sustainability Framework, Baseline Scenario, 2008-2031

(In percent of GDP, unless otherwise indicated)

\begin{tabular}{|c|c|c|c|c|c|c|c|c|c|c|c|c|c|c|c|}
\hline & \multicolumn{3}{|c|}{ Actual } & \multirow[b]{2}{*}{ Average ${ }^{5 /}$} & \multirow[b]{2}{*}{$\begin{array}{r}\text { Standard } \\
\text { Deviation } \\
\end{array}$} & \multicolumn{5}{|l|}{ Estimate } & \multicolumn{5}{|c|}{ Projections } \\
\hline & 2008 & 2009 & 2010 & & & 2011 & 2012 & 2013 & 2014 & 2015 & 2016 & $\begin{array}{l}2011-16 \\
\text { Average } \\
\end{array}$ & 2021 & 2031 & $\begin{array}{l}2017-31 \\
\text { Average } \\
\end{array}$ \\
\hline $\begin{array}{l}\text { Public sector debt } 1 / \\
\mathrm{o} / \mathrm{w} \text { foreign-currency denominated }\end{array}$ & $\begin{array}{l}67.9 \\
44.9\end{array}$ & $\begin{array}{l}68.8 \\
46.3\end{array}$ & $\begin{array}{l}74.9 \\
51.3\end{array}$ & & & $\begin{array}{l}77.7 \\
54.3\end{array}$ & $\begin{array}{l}82.1 \\
59.1\end{array}$ & $\begin{array}{l}85.8 \\
64.6\end{array}$ & $\begin{array}{l}87.4 \\
68.1\end{array}$ & $\begin{array}{l}84.8 \\
68.1\end{array}$ & $\begin{array}{l}82.3 \\
68.1\end{array}$ & & $\begin{array}{l}67.1 \\
55.4\end{array}$ & $\begin{array}{l}49.5 \\
33.6\end{array}$ & \\
\hline Change in public sector debt & -6.0 & 0.9 & 6.0 & & & 2.8 & 4.4 & 3.7 & 1.6 & -2.6 & -2.5 & & -2.4 & -1.7 & \\
\hline Identified debt-creating flows & -2.9 & -1.5 & 9.3 & & & 0.3 & 2.9 & 3.2 & 1.6 & -2.5 & -2.9 & & -2.4 & -1.7 & \\
\hline Primary deficit & -0.3 & 4.8 & 9.0 & 3.3 & 3.0 & 8.1 & 6.6 & 7.0 & 5.6 & 1.9 & 1.4 & 5.1 & 1.4 & 0.4 & 1.2 \\
\hline Revenue and grants & 33.3 & 28.8 & 28.0 & & & 27.6 & 24.8 & 26.4 & 26.9 & 26.8 & 27.3 & & 26.4 & 26.6 & \\
\hline of which: grants & 5.5 & 5.4 & 6.3 & & & 5.2 & 2.5 & 3.4 & 3.5 & 3.0 & 3.0 & & 1.6 & 0.5 & \\
\hline Primary (noninterest) expenditure & 33.0 & 33.5 & 37.0 & & & 35.7 & 31.4 & 33.3 & 32.5 & 28.7 & 28.6 & & 27.7 & 27.1 & \\
\hline Automatic debt dynamics & -1.9 & -6.4 & 0.4 & & & -7.7 & -3.7 & -3.9 & -4.1 & -4.4 & -4.4 & & -3.8 & -2.1 & \\
\hline Contribution from interest rate/growth differential & -4.5 & -2.3 & -3.2 & & & -3.5 & -3.0 & -3.3 & -3.5 & -4.0 & -4.1 & & -3.1 & -1.7 & \\
\hline of which: contribution from average real interest rate & -0.1 & 0.2 & 0.3 & & & 0.1 & 0.8 & 0.8 & 0.9 & 0.5 & 0.3 & & 0.5 & 1.0 & \\
\hline of which: contribution from real GDP growth & -4.3 & -2.4 & -3.5 & & & -3.6 & -3.8 & -4.1 & -4.3 & -4.5 & -4.4 & & -3.6 & -2.7 & \\
\hline Contribution from real exchange rate depreciation & 2.6 & -4.1 & 3.6 & & & -4.1 & -0.7 & -0.6 & -0.7 & -0.5 & -0.3 & & $\ldots$ & $\ldots$ & \\
\hline Other identified debt-creating flows & -0.7 & 0.1 & -0.1 & & & -0.1 & 0.0 & 0.1 & 0.1 & 0.1 & 0.0 & & 0.0 & 0.0 & \\
\hline Privatization receipts (negative) & -0.7 & 0.1 & -0.1 & & & -0.1 & 0.0 & 0.1 & 0.1 & 0.1 & 0.0 & & 0.0 & 0.0 & \\
\hline Recognition of implicit or contingent liabilities & 0.0 & 0.0 & 0.0 & & & 0.0 & 0.0 & 0.0 & 0.0 & 0.0 & 0.0 & & 0.0 & 0.0 & \\
\hline Debt relief (HIPC and other) & 0.0 & 0.0 & 0.0 & & & 0.0 & 0.0 & 0.0 & 0.0 & 0.0 & 0.0 & & 0.0 & 0.0 & \\
\hline Other (specify, e.g. bank recapitalization) & 0.0 & 0.0 & 0.0 & & & 0.0 & 0.0 & 0.0 & 0.0 & 0.0 & 0.0 & & 0.0 & 0.0 & \\
\hline Residual, including asset changes & -3.1 & 2.5 & -3.3 & & & 2.5 & 1.5 & 0.6 & 0.0 & -0.1 & 0.5 & & 0.0 & 0.0 & \\
\hline \multicolumn{16}{|l|}{ Other Sustainability Indicators } \\
\hline PV of public sector debt & $\ldots$ & $\ldots$ & 59.4 & & & 61.6 & 64.7 & 67.1 & 68.0 & 65.9 & 64.0 & & 55.9 & 45.8 & \\
\hline $\mathrm{o} / \mathrm{w}$ foreign-currency denominated & $\ldots$ & $\ldots$ & 35.8 & & & 38.2 & 41.8 & 45.9 & 48.7 & 49.2 & 49.7 & & 44.2 & 29.8 & \\
\hline $\mathrm{o} / \mathrm{w}$ external & & $\ldots$ & 35.8 & & & 38.2 & 41.8 & 45.9 & 48.7 & 49.2 & 49.7 & & 44.2 & 29.8 & \\
\hline $\mathrm{PV}$ of contingent liabilities (not included in public sector debt) & & $\ldots$ & & & & $\ldots$ & & $\ldots$ & $\ldots$ & $\ldots$ & $\ldots$ & & $\ldots$ & & \\
\hline Gross financing need $2 /$ & 2.9 & 7.8 & 11.9 & & & 11.3 & 9.7 & 9.9 & 8.6 & 4.6 & 3.9 & & 4.7 & 4.4 & \\
\hline $\mathrm{PV}$ of public sector debt-to-revenue and grants ratio (in percent) & $\ldots$ & $\ldots$ & 212.3 & & & 223.1 & 260.8 & 254.5 & 252.7 & 245.6 & 234.6 & & 212.0 & 171.8 & \\
\hline $\mathrm{PV}$ of public sector debt-to-revenue ratio (in percent) & $\ldots$ & $\ldots$ & 273.5 & & & 274.8 & 289.5 & 291.9 & 290.9 & 276.3 & 263.6 & & 226.0 & 175.3 & \\
\hline o/w external 3/ & & & 165.0 & & & 170.4 & 186.8 & 199.7 & 208.3 & 206.4 & 204.9 & & 178.7 & 114.1 & \\
\hline Debt service-to-revenue and grants ratio (in percent) $4 /$ & 9.7 & 10.5 & 10.4 & & & 11.8 & 12.6 & 11.3 & 11.0 & 10.3 & 9.2 & & 12.7 & 14.8 & \\
\hline Debt service-to-revenue ratio (in percent) $4 /$ & 11.6 & 12.9 & 13.3 & & & 14.6 & 14.0 & 12.9 & 12.7 & 11.6 & 10.4 & & 13.5 & 15.1 & \\
\hline Primary deficit that stabilizes the debt-to-GDP ratio & 5.7 & 3.8 & 3.0 & & & 5.3 & 2.2 & 3.2 & 4.0 & 4.5 & 3.9 & & 3.8 & 2.1 & \\
\hline \multicolumn{16}{|l|}{ Key macroeconomic and fiscal assumptions } \\
\hline Real GDP growth (in percent) & 6.2 & 3.7 & 5.4 & 6.1 & 2.0 & 5.0 & 5.1 & 5.2 & 5.3 & 5.4 & 5.5 & 5.3 & 5.5 & 5.6 & 5.5 \\
\hline Average nominal interest rate on forex debt (in percent) & 1.1 & 1.1 & 1.1 & 1.2 & 0.2 & 1.4 & 1.5 & 1.4 & 1.5 & 1.3 & 1.3 & 1.4 & 1.7 & 2.9 & 2.1 \\
\hline Average real interest rate on domestic debt (in percent) & 1.2 & 0.6 & 1.8 & 3.3 & 2.6 & 1.8 & 2.3 & 2.6 & 2.6 & 2.5 & 2.6 & 2.4 & 3.7 & 3.6 & 3.5 \\
\hline Real exchange rate depreciation (in percent, + indicates depreciation) & 6.0 & -9.5 & 8.1 & -2.3 & 12.3 & -8.5 & $\ldots$ & $\ldots$ & $\ldots$ & $\ldots$ & $\ldots$ & $\ldots$ & $\ldots$ & $\ldots$ & $\ldots$ \\
\hline Inflation rate (GDP deflator, in percent) & 3.2 & 4.2 & 3.3 & 1.8 & 2.1 & 3.9 & 3.4 & 2.9 & 2.9 & 2.8 & 2.7 & 3.1 & 2.7 & 2.8 & 2.7 \\
\hline Growth of real primary spending (deflated by GDP deflator, in percent) & 0.1 & 0.1 & 0.2 & 0.1 & 0.1 & 0.0 & -0.1 & 0.1 & 0.0 & -0.1 & 0.1 & 0.0 & 0.0 & 0.1 & 0.1 \\
\hline Grant element of new external borrowing (in percent) & $\ldots$ & $\ldots$ & 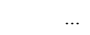 & & $\ldots$ & 29.9 & 31.1 & 31.3 & 31.5 & 31.9 & 31.0 & 31.1 & 10.0 & 5.5 & \\
\hline
\end{tabular}

Sources: Country authorities; and staff estimates and projections.

2/ Gross financing need is defined as the primary deficit plus debt service plus the stock of short-term debt at the end of the last period.

3/ Revenues excluding grants.

4/ Debt service is defined as the sum of interest and amortization of medium and long-term debt.

5/ Historical averages and standard deviations are generally derived over the past 10 years, subject to data availability 
Table 4.Cape Verde: Sensitivity Analysis for Key Indicators of Public Debt 2011-2031

\section{PV of Debt-to-GDP Ratio \\ Baseline}

\section{A. Alternative scenarios}

A1. Real GDP growth and primary balance are at historical averages

A2. Primary balance is unchanged from 2011

A3. Permanently lower GDP growth 1/

A4. Alternative Scenario: Fiscal deterioration

\section{B. Bound tests}

B1. Real GDP growth is at historical average minus one standard deviations in 2012-2013

B2. Primary balance is at historical average minus one standard deviations in 2012-2013

B3. Combination of B1-B2 using one half standard deviation shocks

B4. One-time 30 percent real depreciation in 2012

B5. 10 percent of GDP increase in other debt-creating flows in 2012

\section{PV of Debt-to-Revenue Ratio 2/}

\section{Baseline}

\section{A. Alternative scenarios}

A1. Real GDP growth and primary balance are at historical averages

A2. Primary balance is unchanged from 2011

A3. Permanently lower GDP growth 1/

A4. Alternative Scenario: Fiscal deterioration

62

$\begin{array}{rrrrrrrr}62 & 61 & 59 & 57 & 57 & 57 & 57 & 67 \\ 62 & 66 & 70 & 73 & 78 & 83 & 105 & 152 \\ 62 & 65 & 68 & 69 & 68 & 67 & 64 & 71 \\ 62 & 65 & 69 & 71 & 70 & 70 & 69 & 73\end{array}$

\section{B. Bound tests}

B1. Real GDP growth is at historical average minus one standard deviations in 2012-2013

B2. Primary balance is at historical average minus one standard deviations in 2012-2013

B3. Combination of B1-B2 using one half standard deviation shocks

B4. One-time 30 percent real depreciation in 2012

B5. 10 percent of GDP increase in other debt-creating flows in 2012

$\begin{array}{llllllll}223 & 245 & 224 & 213 & 212 & 208 & 219 & 253 \\ 223 & 267 & 265 & 272 & 290 & 303 & 399 & 571 \\ 223 & 262 & 257 & 258 & 253 & 244 & 241 & 265 \\ 223 & 263 & 268 & 274 & 277 & 276 & 288 & 319\end{array}$

$\begin{array}{llllllll}223 & 264 & 262 & 262 & 256 & 247 & 233 & 207 \\ 223 & 259 & 251 & 249 & 242 & 231 & 209 & 169 \\ 223 & 253 & 239 & 238 & 231 & 221 & 200 & 163 \\ 223 & 321 & 305 & 297 & 288 & 275 & 252 & 224 \\ 223 & 304 & 294 & 290 & 283 & 271 & 245 & 198\end{array}$

Debt Service-to-Revenue Ratio 2/

$\begin{array}{ll}66 & 69 \\ 64 & 66 \\ 63 & 63 \\ 80 & 80 \\ 75 & 78\end{array}$

$\begin{array}{lllll}71 & 69 & 67 & 61 & 55 \\ 67 & 65 & 63 & 55 & 45 \\ 64 & 62 & 60 & 53 & 43 \\ 80 & 77 & 75 & 66 & 60 \\ 78 & 76 & 74 & 65 & 53\end{array}$

\section{2}

\section{Baseline}

$\begin{array}{rrrrrrrr}12 & 13 & 10 & 9 & 5 & 1 & 12 & 33 \\ 12 & 13 & 12 & 12 & 13 & 14 & 47 & 103 \\ 12 & 13 & 11 & 11 & 11 & 10 & 16 & 32 \\ 12 & 13 & 12 & 12 & 11 & 10 & 15 & 21\end{array}$

A1. Real GDP growth and primary balance are at historical averages

A2. Primary balance is unchanged from 2011

A3. Permanently lower GDP growth $1 /$

A4. Alternative Scenario: Fiscal deterioration

\section{B. Bound tests}

B1. Real GDP growth is at historical average minus one standard deviations in 2012-2013

B2. Primary balance is at historical average minus one standard deviations in 2012-2013

B3. Combination of B1-B2 using one half standard deviation shocks

B4. One-time 30 percent real depreciation in 2012

B5. 10 percent of GDP increase in other debt-creating flows in 2012

$\begin{array}{rrrrrrrr}12 & 13 & 11 & 11 & 11 & 10 & 16 & 22 \\ 12 & 13 & 11 & 11 & 10 & 8 & 12 & 14 \\ 12 & 13 & 11 & 10 & 8 & 5 & 9 & 12 \\ 12 & 14 & 14 & 14 & 14 & 14 & 23 & 35 \\ 12 & 13 & 13 & 13 & 22 & 21 & 21 & 22\end{array}$

Sources: Country authorities; and staff estimates and projections.

1 / Assumes that real GDP growth is at baseline minus one standard deviation divided by the square root of the length of the projection period.

2/ Revenues are defined inclusive of grants. 
Figure 1. Cape Verde: Indicators of Public and Publicly Guaranteed External Debt under Alternatives Scenarios, 2011-2031 1/
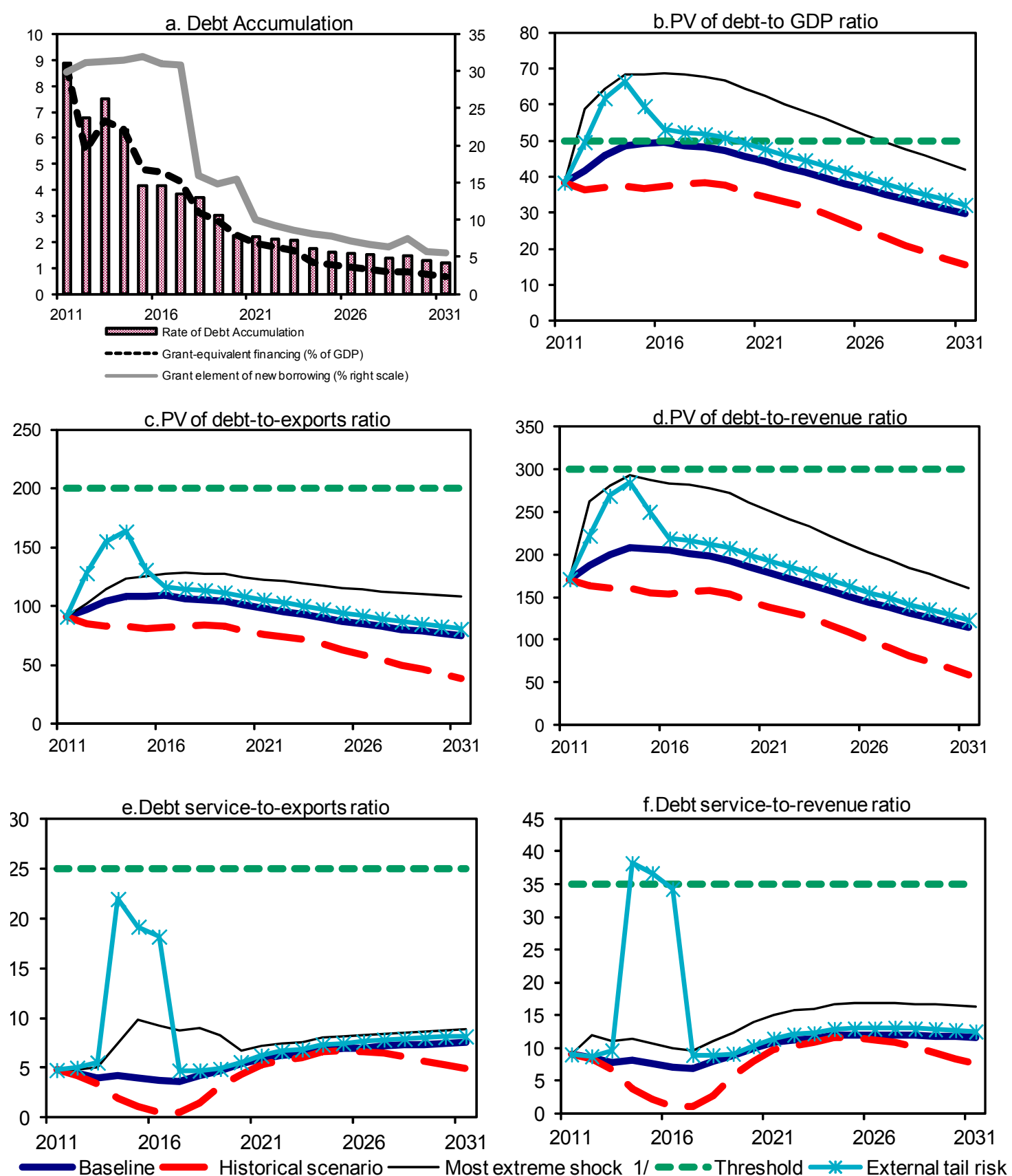

Sources: Country authorities; and staff estimates and projections.

1/ The most extreme stress test is the test that yields the highest ratio in 2021. In figure b. it corresponds to a One-time depreciation shock; in c. to a Terms shock; in d. to a One-time depreciation shock; in e. to a Exports shock and in figure f. to a One-time depreciation shock 
Figure 2.Cape Verde: Indicators of Public Debt Under Alternative Scenarios, 2011-2031 1/
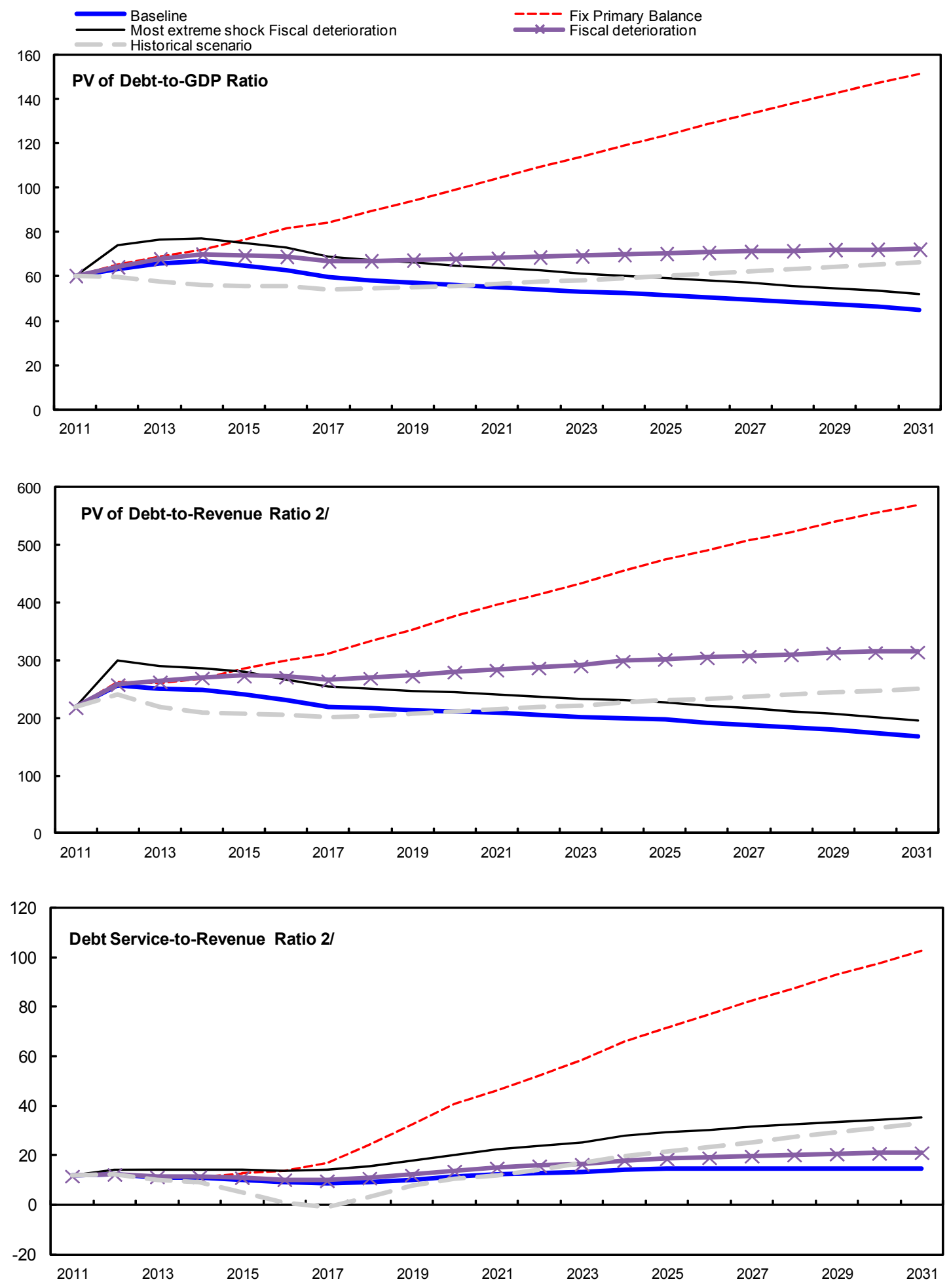

Sources: Country authorities; and staff estimates and projections.

1/ The most extreme stress test is the test that yields the highest ratio in 2021

2/ Revenues are defined inclusive of grants. 
ANNEX I-CAPE VERDE: RELATIONS WITH THE FUND

(as of November 30, 2011)

I. Membership Status: Joined: November 20, 1978;

Article VIII

II. General Resources Account:

SDR Million

Quota

9.60

\%Quota

Fund holdings of currency

9.59

100.00

Reserve tranche position

III. SDR Department:

0.02

99.93

Net cumulative allocation

SDR Million

9.17

0.17

Holdings

5.36

\%Allocation

100.00

IV. Outstanding Purchases and Loans:

SDR Million

ECF arrangements

4.21

58.51

\%Quota

43.81

V. Latest Financial Arrangements:

\begin{tabular}{lcrrr}
\hline Type & $\begin{array}{r}\text { Date of } \\
\text { Arrangement }\end{array}$ & $\begin{array}{r}\text { Expiration } \\
\text { Date }\end{array}$ & $\begin{array}{r}\text { Amount } \\
\text { Approved } \\
\text { (SDR Million) }\end{array}$ & $\begin{array}{r}\text { Amount } \\
\text { Drawn } \\
\text { (SDR Million) }\end{array}$ \\
\hline ECF $^{1 /}$ & Apr 10, 2002 & Jul 31, 2005 & 8.64 & 8.64 \\
Stand-By & Feb 20, 1998 & Mar 15, 2000 & 2.50 & 0.00 \\
${ }^{1 /}$ Formerly PRGF & & & & \\
\hline
\end{tabular}

VI. Projected Payments to Fund ${ }^{2 !}$

(SDR Million; based on existing use of resources and present holdings of SDRs):

\begin{tabular}{lccccc}
\hline & \multicolumn{5}{c}{ Forthcoming } \\
\cline { 2 - 6 } & 2011 & 2012 & 2013 & 2014 & 2015 \\
\cline { 2 - 6 } Principal & 0.37 & 1.61 & 1.24 & 0.74 & 0.25 \\
Charges/interest & & 0.01 & 0.01 & 0.01 & 0.01 \\
Total & 0.37 & 1.62 & 1.25 & 0.75 & 0.26 \\
\hline
\end{tabular}

${ }^{2 /}$ When a member has overdue financial obligations outstanding for more than three months, the amount of such arrears will be shown in this section. 
VII. Implementation of HIPC Initiative: Not Applicable

VIII. Implementation of Multilateral Debt Relief Initiative (MDRI): Not Applicable

\section{Safeguards Assessments}

Two safeguards assessments of the Bank of Cape Verde (BCV) were completed in 2002 and 2008. The 2008 assessment was conducted voluntarily at the request of the authorities with respect to the Policy Support Instrument (PSI). It concluded that the BCV had adopted some of the measures recommended in 2002, but also made new recommendations to address further safeguards vulnerabilities notably in the internal audit mechanism, system of internal controls, and transparency practices. While the implementation of certain safeguards recommendations remains work in progress, the $\mathrm{BCV}$ has since improved the completion of the audits and the publication of the audited financial statements on its website.

\section{Exchange Arrangements}

The de facto and de jure exchange rate arrangement of Cape Verde is a conventional fixed peg. The escudo has been pegged to the euro at a rate of CVE 110.265 per euro since January 4, 1999. Cape Verde accepted the obligations of Article VIII of the Articles of Agreement effective July 1, 2004. It maintains an exchange system that is free of restrictions on the making of payments and transfers for current international transactions.

\section{Previous Article IV Consultation and PSI Reviews}

The Executive Board concluded the Article IV consultation and approved the request for a PSI on July 31, 2006 (Country Report No. 06/334); the first PSI review on January 19, 2007 (Country Report No. 07/44); the second PSI review on May 7, 2007 (Country Report No. 07/223); the third PSI review on December 21, 2007 (Country Report No. 08/37); the 2008 Article IV consultation and the fourth PSI review on June 30, 2008 (Country Report No. 08/248); the fifth PSI review on December 22, 2008 (Country Report No. 09/14); the sixth PSI review on June 19, 2009 (Country Report No. 09/198); the seventh PSI review on December 18, 2009 (Country Report No. 09/328); and the eight PSI review on July 19, 2010 (Country Report No. 10/218). The Executive Board concluded the 2010 Article IV consultation and approved a 15-month PSI arrangement in November 2010 (Country Report No. 10/349). The executive board concluded the first review under the PSI on a lapse-of-time basis on July 27, 2011 (Country Report No. 11/254).

\section{Technical Assistance}

Since 1985, Cape Verde has received substantial technical assistance. Technical assistance activities since 2004 are listed below: 


\section{Fiscal Affairs Department (FAD):}

- June 2004: help the authorities move to a VAT, rationalize the import tariff, and overhaul the domestic indirect tax system.

- $\quad$ October 2004: review tax administration, including VAT implementation and help assess tax exemptions and incentives.

- $\quad$ September 2005: assess tax exemptions and incentives.

- June 2008: review and rationalize tax exemptions.

\section{Statistics Department (STA):}

- $\quad$ November 2003, January 2006: National accounts.

- $\quad$ February 2004: Balance of payments statistics.

- $\quad$ March 2004, April 2006, February 2007, August 2008: Government finance statistics.

- June 2004, May 2006, October 2007: Price statistics.

- $\quad$ March 2007: Monetary statistics and reporting.

\section{Monetary and Capital Markets (MCM)}

- April 2004: Accounting, financial sector regulation, monetary operations and liquidity management.

- $\quad$ November 2005, March 2006, June 2006, November 2006, and July 2007: Banking supervision, liquidity management, exchange regime and reserves management.

- $\quad$ March 2008: Macro-prudential indicators.

- $\quad$ November 2008, January 2009, and March 2009: Financial Sector Assessment Program (FSAP), and various follow up missions on banking supervision.

- $\quad$ February 2009 and September 2009: Joint IMF-World Bank on debt management (DeMPA) and on Medium-Term Debt Strategy (MTDS).

- December 2010: Liquidity management and monetary policy operations.

- June 2011: Mid-term Debt Strategy Development Follow-up.

- $\quad$ October 2011: Enhancing Banking Supervision and Crisis Resolution.

\section{Legal (LEG):}

- $\quad$ October 2006 and March 2008: Tax legislation.

- $\quad$ March 2007: AML/CFT initial assessment, and legal drafting on March 2008.

XIII. Resident Representative: None. 
ANNEX II-Cape Verde: Joint IMF-World Bank Management ACTION PlanIMPLEMENTATION MATRIX

\begin{tabular}{|c|c|c|c|}
\hline Title & Products & $\begin{array}{l}\text { Provisional } \\
\text { Timing of } \\
\text { Missions }\end{array}$ & $\begin{array}{c}\text { Expected Delivery } \\
\text { Date }\end{array}$ \\
\hline \multicolumn{4}{|c|}{ A. Mutual information on relevant work programs } \\
\hline $\begin{array}{l}\text { Bank work } \\
\text { program in the } \\
\text { next } 12 \\
\text { months }\end{array}$ & $\begin{array}{l}\text { Lending (new operations): } \\
\text { - Energy Operation (IBRD) } \\
\text { - PRSC-7 (IDA) } \\
\text { - PRSC-8 (IDA) } \\
\text { - Trust Fund for Strengthening } \\
\text { Capacity in M\&E System (PRLP) - Grant } \\
\text { Analytical and advisory activities: } \\
\text { - Higher Education ESW } \\
\text { - PEMFAR } \\
\text { - Country Economic Memorandum } \\
\text { - Inter-Island Transportation Study } \\
\text { - Public Investment management Non- } \\
\text { - General economic monitoring }\end{array}$ & $\begin{array}{l}\text { Preparation } \\
\text { completed } \\
\text { Jan. } 2012 \\
\text { May 2012, Sept. } \\
2012 \\
\text { March, } 2012 \\
\text { Preparation } \\
\text { completed } \\
\text { Dec. } 2012 \\
\text { Feb. } 2012 \\
\text { Apr. } 2012 \\
\text { March } 2012 \\
2009 \\
\text { May, } 2012\end{array}$ & $\begin{array}{l}\text { Q3, FY12 (Jan. 2012) } \\
\text { Q3, FY12 } \\
\text { Q4, FY12 (Apr. 2012) } \\
\text { Q1, FY13 } \\
\text { Q2, FY13 } \\
\text { Q3, FY12; Q2, FY13 }\end{array}$ \\
\hline
\end{tabular}




\begin{tabular}{|l|l|l|l|}
\hline Title & Products & \multicolumn{1}{c|}{$\begin{array}{c}\text { Provisional Timing of } \\
\text { Missions }\end{array}$} & $\begin{array}{c}\text { Expected Delivery } \\
\text { Date }\end{array}$ \\
\hline \multicolumn{3}{|c|}{ B. Mutual information on relevant work programs } \\
\hline $\begin{array}{l}\text { IMF work program in } \\
\text { next 12 months }\end{array}$ & Staff visit & May-June 2012 & \\
\cline { 2 - 4 } & Article IV & September/October 2012 & $\begin{array}{l}\text { Board meeting } \\
\text { December } 2012\end{array}$ \\
\hline \multicolumn{5}{|c|}{ C. Agreement on joint products and missions } \\
\hline JSAN on GPRSP III & JSAN & September, 2012 & Q2, FY13 \\
\hline
\end{tabular}

CInternational Monetary Fund. Not for Redistribution 


\section{ANNEX III-CAPE VERDE: STATISTICAL IsSUES}

1. Data provision has some shortcomings, but is broadly adequate for surveillance. There is a need for substantial improvements in the areas of national accounts, financial sector, and balance of payments. The country has participated in the GDDS since February 2004. Cape Verde's plans for improvements of its statistical system are posted on the Fund's DSBB; the metadata of the monetary and financial sector were updated in August 2007. The country has benefited from STA technical assistance under the GDDS project for Lusophone Africa.

2. The authorities are taking steps to strengthen statistics, but the statistical system still suffers from a shortage of financial and human resources. A comprehensive master plan has been developed under the direction of the National Statistical Institute (INE), which assesses the need for upgrading the agencies that constitute the statistical system and outlines steps to broaden and improve all areas of statistics.

\section{Real Sector Statistics}

3. Although some improvements have been made to the national accounts, significant weaknesses remain, including a lack of reliable demand-side GDP. The timeliness of the data has not improved, with the INE releasing production and expenditurebased GDP data for 2005-07 in 2010 and providing production-based GDP estimates for 2008 to the AFR mission in September 2010. However, the lag is still lengthy by international standards. INE is receiving technical assistance from Brazil and Spain in developing business indicators and national accounts. On May 2011, INE signed a cooperation protocol with its Spanish counterpart to enhance national accounts and produce environmental statistics. INE plans to publish quarterly GDP data by 2012.

4. INE is currently working on a complete overhaul of the national accounts. It is updating the national accounts benchmark and base years from 1980 to 2002 and adopting the 1993 SNA. The objective is timely compilation of GDP by industry and expenditure categories at current and constant prices as well as institutional sector accounts.

5. INE will also introduce tourism satellite accounts. These accounts will measure upstream linkages between the tourism sector and the rest of the economy and the import content of tourism exports.

6. Full implementation of the $1993 S N A$ would require a substantial improvement in source data collection, for which capacity is currently lacking. The 2006 STA mission found a critical need to improve the timeliness and accuracy of national accounts source data, in particular the business survey. The capacity of INE staff working on national accounts was found to be overstretched; they assess in detail and correct individual source data entriessomething not undertaken in most countries by national accounts compilers. The statistical master plan will address many of these challenges. 
7. A revamped CPI with new methodology was launched in February 2008. The previous official CPI was based on weights dating back to 1989, four years before imports were liberalized. Import liberalization considerably changed consumption patterns. INE has had assistance from the National Statistics Institute of Portugal and benefited from STA CPI missions in May-June, 2006, October 2006, and October 2007, to support the introduction and dissemination of the revised CPI. The new index has new weights and an updated commodity basket.

8. INE publishes labor market statistics only on annual basis with long delays. Recently, it published annual unemployment data, which is comparable to EUROSTAT and AFRISTAT. INE recently published the 2010 census. There is no data on wages in different sectors. The INE staff are receiving training from Senegal on total factor productivity (TFP) statistics of companies that hire regular employees, but they are also in need of technical assistance on TFP statistics in other sectors of the economy, such as public administration, agriculture, and companies that only hire temporary contactors instead of regular employees.

\section{Government Finance Statistics}

9. The fiscal data have been improved. Benefiting from TA under the GDDS project for Lusophone Africa, the Government Finance Statistics (GFS) compilation system is being upgraded. Most deposits previously held with commercial banks have been consolidated at the central bank. SIGOF, the Integrated Online Budget Management System, has been expanded to cover all semi-autonomous institutes and most municipalities by the end of 2007. The authorities have started reporting GFS for publication in the GFS Yearbook.

\section{However, quality is a concern. The fiscal accounts are subject to statistical} discrepancies, and flows and stocks are not always consistent. A significant delay in donor reporting of project financing also affects the accuracy of fiscal data. Despite the recent revision of external debt data, weaknesses regarding the public and publically guaranteed debt of state owned enterprises persist.

\section{Monetary and Financial Statistics}

\section{The monetary and financial statistics is adequate, but the quality of monetary} survey could be further improved. An STA mission undertaken in March 2007 helped the Banco de Cabo Verde (BCV) to finalize the standardized report forms (SRFs) for reporting monetary statistics to STA. SRF-based monetary data have been published in the IFS Supplement since June 2007. These data are fully aligned with the recommendations of the Monetary and Financial Statistics Manual. The integrated monetary database that meets STA, AFR, and BCV statistical needs is now in place. However, there are presentation gaps between the monetary survey generated by the STA and the one produced by the BCV, which the BCV indicates can be resolved in a short period of time. 


\section{External Sector Statistics}

12. The accuracy, periodicity, and timeliness of balance of payments statistics compiled by BCV have continued to improve. A greater use of surveys, combined with the International Transactions Reporting System implemented by the BCV, has permitted a significant expansion of data sources and statistical coverage, which to a large extent follow the recommendations of the 5th edition of the Balance of Payments Manual. However, a lack of data from one bank on external flows remains. Dissemination of quarterly BOP data on the $\mathrm{BCV}$ website has been regular and in 2007 the BCV resumed regular and timely transmission of these data to STA for publication in International Financial Statistics and in the Balance of Payments Statistics Yearbook. 


\section{Cape Verde: Common Indicators Required for Surveillance}

(As of December 11, 2011)

\begin{tabular}{|c|c|c|c|c|c|}
\hline & $\begin{array}{c}\text { Date of } \\
\text { Latest } \\
\text { Observation }\end{array}$ & $\begin{array}{l}\text { Date } \\
\text { Received }\end{array}$ & $\begin{array}{l}\text { Frequency } \\
\text { of Data }^{7}\end{array}$ & $\begin{array}{l}\text { Frequency } \\
\quad \text { of } \\
\text { Reporting }^{7}\end{array}$ & $\begin{array}{l}\text { Frequency } \\
\quad \text { of } \\
\text { Publication }^{7}\end{array}$ \\
\hline Exchange Rates & 09/30/11 & $11 / 06 / 11$ & $\mathrm{D}$ & $\mathrm{D}$ & $\mathrm{D}$ \\
\hline $\begin{array}{l}\text { International Reserve Assets } \\
\text { and Reserve Liabilities of the } \\
\text { Monetary Authorities }{ }^{1}\end{array}$ & $09 / 30 / 11$ & $11 / 06 / 11$ & $\mathrm{D}$ & W & M \\
\hline Reserve/Base Money & $09 / 30 / 11$ & $11 / 06 / 11$ & $\mathrm{D}$ & W & M \\
\hline Broad Money & $09 / 30 / 11$ & $11 / 06 / 11$ & M & M & M \\
\hline Central Bank Balance Sheet & $09 / 30 / 11$ & $11 / 06 / 11$ & $\mathrm{D}$ & W & $\mathrm{M}$ \\
\hline $\begin{array}{l}\text { Consolidated Balance Sheet of } \\
\text { the Banking System }\end{array}$ & $09 / 30 / 11$ & $11 / 06 / 11$ & M & M & M \\
\hline Interest Rates ${ }^{2}$ & $09 / 30 / 11$ & $11 / 06 / 11$ & M & M & M \\
\hline Consumer Price Index & $10 / 30 / 11$ & $11 / 15 / 11$ & $\mathrm{M}$ & $\mathrm{M}$ & M \\
\hline $\begin{array}{l}\text { Revenue, Expenditure, Balance } \\
\text { and Composition of Financing }{ }^{3}- \\
\text { Central Government }\end{array}$ & $09 / 30 / 11$ & 09/30/11 & Q & Q & Q \\
\hline $\begin{array}{l}\text { Stocks of Central Government } \\
\text { and Central Government- } \\
\text { Guaranteed Debt }^{5}\end{array}$ & 09/30/11 & $09 / 30 / 11$ & Q & Q & $A$ \\
\hline $\begin{array}{l}\text { External Current Account } \\
\text { Balance }\end{array}$ & $09 / 30 / 11$ & $11 / 15 / 11$ & Q & Q & Q \\
\hline $\begin{array}{l}\text { Exports and Imports of Goods } \\
\text { and Services }\end{array}$ & $09 / 30 / 11$ & $11 / 15 / 11$ & Q & Q & Q \\
\hline GDP/GNP & 2008 & Sept. 2010 & A & A & A \\
\hline Gross External Debt & $09 / 30 / 11$ & $09 / 30 / 11$ & $Q$ & $Q$ & A \\
\hline $\begin{array}{l}\text { International Investment } \\
\text { Position }\end{array}$ & 2007 & Aug. 2008 & $A$ & $A$ & $A$ \\
\hline
\end{tabular}

${ }^{1}$ Includes reserve assets pledged or otherwise encumbered as well as net derivative positions.

${ }^{2}$ Both market-based and officially-determined, including discount rates, money market rates, rates on treasury bills, notes and bonds.

${ }^{3}$ Foreign, domestic bank, and domestic nonbank financing.

${ }^{4}$ The general government consists of the central government (budgetary funds, extra budgetary funds, and social security funds) and state and local governments.

${ }^{5}$ Including currency and maturity composition.

${ }^{6}$ Includes external gross financial asset and liability positions vis-à-vis nonresidents.

${ }^{7}$ Daily (D), weekly (W), monthly (M), quarterly (Q), annually (A), irregular (I); and not available (NA). 
January 30, 2012

\section{IMF Executive Board Completes Second and Final Review of Cape Verde's Performance under Policy Support Instrument}

The Executive Board of the International Monetary Fund (IMF) today completed the second and final review of Cape Verde's economic performance under the 15-month Policy Support Instrument $^{1}$ (PSI) program approved on November 2010 (see Press Release No. 10/457). In completing the review, the Executive Board granted waivers of nonobservance of assessment criteria.

Following the Executive Board's discussion of Cape Verde, Ms. Nemat Shafik, Deputy Managing Director and Acting Chair, made the following statement:

"The Cape Verde authorities tightened macroeconomic policies in the second half of 2011 in response to risks to macroeconomic stability arising from international commodity price increases and the European debt crisis. Given lingering global uncertainties, policy restraint will be needed in the coming months to safeguard the exchange rate peg and build buffers against shocks.

"The authorities plan to reduce the budget deficit and external debt over the medium term to strengthen the fiscal position and support private sector growth. They will need to increase domestic revenue, contain current spending, and proceed cautiously on capital spending while relying on concessional borrowing as much as possible.

\footnotetext{
${ }^{1}$ The IMF's framework for PSIs is designed for low-income countries (and small island states) that may not need IMF financial assistance, but still seek close cooperation with the IMF in preparation and endorsement of their policy frameworks. For more details see PSI Factsheet at http://www.imf.org/external/np/exr/facts/psi.htm.
} 
"The recent increase in the Bank of Cape Verde's policy rate and the reserve requirements for commercial banks will help contain inflation and bolster international reserves. Growing financial sector vulnerabilities call for further improving banking regulation and supervision. The recent establishment of a financial stability committee represents a welcome step in this direction.

"While progress has been made toward the Millennium Development Goals, unemployment remains high. This calls for reforms to enhance competitiveness, diversify the economy, and improve the functioning of the labor market. Faster progress in restructuring state-owned enterprises will also be important." 


\section{Statement by Mr. Kossi Assimaidou, Executive Director for Cape Verde January 30, 2012}

Mr. Assimaidou submitted the following statement:

1. Cape Verde's current PSI program has ambitioned to consolidate macroeconomic and financial stability and support the exchange rate peg. To this end, it has sought to promote continued prudence in public debt and public finance management, build up international reserves, and nurture financial sector stability. Most notably, the PSI has been instrumental in supporting the authorities' policy and reform agenda.

2. During the implementation of the successive PSIs in recent years, major strides were made in stimulating growth and reducing poverty. In parallel, data reported in Cape Verde's latest PRSP progress report and census highlight encouraging unemployment trends in recent years. However, measuring the significant inroads made in reducing unemployment among the Cape Verdean population remains challenging, notably in light of the change in computational methods following the adoption of ILO measurement standards and the substantial import of labor from West African countries in recent years.

3. More recently, the Cape Verdean economy faced large external shocks that stem notably from the turmoil in the euro area and increases in global food and fuel prices. As a result, some macroeconomic fundamentals and performance under the program were adversely affected. In response to these developments, the authorities promptly took necessary policy actions, on both the fiscal and monetary sides, to mitigate the negative impact of these shocks. While the timely response of the authorities has been instrumental in sustaining economic growth and safeguarding macroeconomic and financial stability, policy challenges remain daunting and could become even more so in the event of further deterioration of the external environment. The authorities will continue to address these challenges in a timely manner and with IMF's advice.

\section{Recent Economic Developments and Program Performance}

4. Preliminary estimates suggest that growth slowed down slightly in 2011 to reach about 5 percent, as the external environment deteriorated largely on account of the Eurozone crisis. While tourism and public investment largely maintained their contribution to growth, FDI declined significantly and official transfers were lower than expected. Higher global food and fuel prices fueled inflation and contributed to widening the current account deficit. Reflecting these unfavorable circumstances, the external position of the country weakened, with a notable decline in international reserves. Delayed disbursement of donor assistance aggravated these reserve losses by leading to a reduction of government deposits at the $\mathrm{BCV}$.

5. Performance under the fiscal program was satisfactory as all quantitative targets for end-September 2011 were met. However, on the monetary front, the two quantitative criteria 
related to net domestic assets (NDA) and net international reserves (NIR) were missed owing largely to deteriorating external conditions, particularly in the euro area.

6. To mitigate the adverse impact of external developments, the authorities were able to strengthen the external position in the second half of 2011 through a tighter fiscal and monetary policy stance. On the fiscal side, the deficit was kept under budgeted levels, as the authorities took steps to significantly cut spending and sustain strong tax revenue performance. A number of measures were adopted that notably aimed to increase the efficiency of public administration, streamline public agencies, and ensure stricter control over the wage bill for civil servants. At the same time, there was limited recourse to domestic budget financing.

7. On the monetary side, the BCV broadened the scope of reserve requirements in July 2011 to include government deposits. In parallel, it took necessary steps to constrain the growth of credit as well as reserve and broad money notably through the resumption of openmarket operations. As a result of the abovementioned policy measures, year-end preliminary estimates show that reserve losses were contained and the growth of net domestic assets of the central bank was significantly curtailed.

8. In view of the corrective actions taken, the authorities are requesting waivers of nonobservance of end-September 2011 assessment criteria related to net domestic assets and net international reserves.

9. On the structural front, program performance was strong with five out of seven benchmarks set for the review being met, including the signing of memoranda on fiscal and monetary coordination and the setup of a financial stability committee; the establishment of a framework for the issuance of fungible treasury bonds; the establishment of a medium-term debt strategy, and the inclusion of cross-debt between state-owned enterprises in the report on contingent liabilities. Work toward completing the missed structural benchmarks which entail the submission of the draft income tax bill and new banking law to the parliament is underway and expected to be completed early in 2012 .

\section{Policies for 2012 and Onwards}

10. As indicated above, the authorities promptly took actions in the face of the weakening external environment to mitigate the subsequent adverse impact on macroeconomic fundamentals. In light of Cape Verde's exposure to the current Eurozone crisis, they will remain vigilant, standing ready to take necessary actions to cope with external shocks.

11. In this connection, it is the authorities' intention to keep the stance of fiscal and monetary policies tight so as to preserve fiscal and debt sustainability and safeguard the external position. 
12. On the fiscal front, the authorities underscored their medium-term objective of containing the fiscal deficit and minimizing recourse to domestic financing. In the short run, they plan to keep a tight control over public spending. However, care will be taken to implement austerity measures in consultation with social partners with a view to preserving social stability, sustaining economic growth, and securing further progress toward poverty reduction.

13. The authorities agree that the existing social safety net provides good protection to the poor and the vulnerable, as noted in the staff report. Under these circumstances, they believe that any proposed change in the social safety net should be supported by a comprehensive analysis so as not to jeopardize its adequacy. In this respect, they are of the view that more work needs to be conducted to support staff's proposal to reduce the social security fund's tax rate on wage income and restructure the benefit side to ensure the fund's long-term fiscal solvency. They are concerned that the implementation of such measures would ultimately contribute to reversing hard-won gains in poverty reduction.

14. On its part, the BCV has continued to tighten the stance of monetary policy. Earlier this month, the central bank raised the policy rate and reserve requirements on deposit liabilities, thereby increasing the prospects for further improvements in monetary aggregates and the balance of payments. At this current juncture, keeping the monetary policy stance tight will help achieve the key immediate monetary policy goal which is to stabilize reserve coverage at around 3 months of imports and thus protect the peg.

15. While the fiscal and monetary authorities will work toward achieving their respective policy goals, they will also continue to attach great importance to further strengthening monetary and fiscal policy coordination. Such coordination was tellingly illustrated by the concerted actions taken recently by the fiscal and monetary authorities to support monetary adjustment and strengthen the external position.

16. In view of the risks to financial sector stability stemming from domestic and external factors, strict supervision and regulation of the financial sector will continue to be exercised. At the same time, ongoing efforts to strengthen the supervisory and regulatory framework for onshore and offshore banks will be pursued and adequate use of the newly-established financial stability committee will be made.

17. The authorities see merit in developing a consolidated view of public accounts, integrating the social security fund (INPS), local administrations, and state-owned enterprises (SOEs). SOE reform will be carried forward, notably with a view to generating more efficiency gains in the functioning of public administration. In particular, work is underway to improve the financial situation of the electricity and water company (Electra) and airline company (TACV). In this connection, substantial progress has already been made in recent months in reforming Electra, including the adoption of an action plan for the second phase of Electra's institutional restructuring, the design of a comprehensive approach to the financial 
reform of Electra, the adoption of a new regulatory tariff-adjustment model, and the signature of a results-based management contract between Electra and the government of Cape Verde. ${ }^{1}$

18. In conclusion, I would like to reiterate that the authorities highly value the advice they have received from the IMF in the context of the successive PSIs and they continue to attach great importance to maintaining a close relationship with the IMF.

19. In light of Cape Verde's broadly satisfactory performance under the Policy Support Instrument, I would appreciate Directors' support for the conclusion of the second review of Cape Verde's PSI program.

\footnotetext{
${ }^{1}$ See Cape Verde-Recovery and Reform of the Electricity Sector Project, the World Bank, January 2011.
} 